\title{
A PROBABILITY APPROACH TO THE HEAT EQUATION
}

\author{
BY \\ J. L. DOOB
}

0. Introduction. Let $u$ be a function whose domain is some open set in a Euclidean space. Then, under various conditions on $u$, there is a well defined first boundary value (Dirichlet) problem, involving an analysis of the boundary points of the domain, and a more or less closely associated problem of the existence of boundary limits of $u$. The evaluation of $u$ in terms of the boundary function thus obtained leads back to the Dirichlet problem. These problems have been studied most intensively for harmonic functions, and lead to problems in potential theory and to the theory of subharmonic and superharmonic functions.

To the probabilist, the most natural way to study these problems is by means of stochastic processes of diffusion type. This means that the probabilist lumps together parabolic and elliptic partial differential equations. At first this may seem unnatural to the analyst, since the general Dirichlet problem is not ordinarily studied for parabolic equations, but we shall see that, in fact, the probability approach makes the setting, and solution, of the Dirichlet problem just as natural for parabolic as for elliptic equations. In a previous paper, [2], an elliptic equation, Laplace's equation, was studied from a probability point of view. In the present paper we study a parabolic equation, the heat equation, from this point of view. Just as in the case of Laplace's equation, we find that the key questions are tied up with the properties of the solutions along certain probability paths, and that the sub and super functions, introduced by Perron in his study of the Dirichlet problem for Laplace's equation, are fundamental tools. Just as the discontinuous subharmonic functions cause some difficulty in the one study, so the discontinuous subparabolic functions cause difficulty in the present one. In both cases, the discontinuous functions are, however, well behaved on certain probability paths.

As would be expected, a detailed approach to these classical partial differential equations from a modern probability point of view is essentially a real variable approach. In the case of Laplace's equation, the real variable approach to the Dirichlet problem, boundary value problems, and potential theory, is well known, although much of the work is of very recent date. Since there are almost no results in this direction available in the literature of the heat equation, the logical order of development in this paper differs from that in [2]. However the methods and results are surprisingly similar, and some proofs are omitted or only sketched here, since they are too similar to those

Received by the editors January 20, 1955. 
in [2] to be reproduced in detail. There are of course essential differences in the significance of the results, in spite of the formal similarity in the statements of the theorems. In fact, all the variables in Laplace's equation play the same $\mathrm{r}$ le, whereas the time and space variables in the heat equation play essentially different roles. The point is, however, that in both cases there is a certain family of paths, Brownian trajectories, defined to match the equation, but the same for all domains, along which the functions have simple continuity properties and have boundary limits. The Dirichlet problem in each case is solved by setting the value of the function at a point $z$ of its domain equal to the average value of the boundary function at the first boundary point met by a Brownian trajectory from $z$. An irregular boundary point is characterized by the fact that almost all Brownian trajectories from the point enter the domain at once, staying there for some time interval, and irregular boundary points are irrelevant to Dirichlet solutions because almost no Brownian trajectory from a point of a domain first meets the boundary in an irregular point. Thus the main difference in results between Laplace's equation and the heat equation lies in the type of Brownian trajectory involved. Most of the theorems read the same way. (However the class of irregular boundary points causes more difficulties in the case of the heat equation, and there are still unsolved problems here.) For a more general elliptic or parabolic equation, there would be corresponding theorems, involving corresponding probability paths, but of course the regularity properties of the coefficients would cause complications.

For further work on Laplace's equation and the heat equation, from a probabilistic point of view, stressing a detailed study of the relevant Green's functions, see a forthcoming paper by G. A. Hunt in these Transactions.

From the point of view of the nonprobabilist, for whom the interrelations between the theories of probability and heat have no intrinsic interest, we can describe the results of this paper as follows. The Dirichlet problem for the heat equation on an arbitrary open set is solved, and the regular and irregular boundary points are characterized. The general theorems on the existence of boundary limits of harmonic and subharmonic functions, due to Fatou, Littlewood, Privalov, are extended to parabolic and subparabolic functions with arbitrary domains of definition. It is shown that the most natural curves of approach to the boundary along which such functions have limits are certain probability trajectories which do not depend on the domains in question.

1. Subparabolic and parabolic functions. Throughout this paper, we shall deal with functions defined on subsets of Euclidean $N+1$ space, with $N \geqq 1$, denoting a point by $z:\left(\xi_{1}, \cdots, \xi_{N}, s\right)$. We define $\operatorname{ord}(z)=s$, and describe $z_{1}$ as below $z_{2}$ if ord $\left(z_{1}\right)<\operatorname{ord}\left(z_{2}\right)$. A point will be said to be below a set if it is below each point of the set, and a set will be said to be bounded from below if there is a point below it. A point set all of whose points have the same ord value will be said to be horizontal. Addition and subtraction of points is to be 
interpreted in terms of the corresponding operations on the individual coordinates. The distance from $z$ to the origin will be denoted by $|z|$. A single point $\infty$ is adjoined to the $N+1$ space. A neighborhood of this point is to be any point set including it and all finite points below some horizontal hyperplane. The point $\infty$ will be said to be below all finite points. A point set will be said to be finite if it does not contain the point $\infty$. There should be no confusion with sets whose points are finite in number. Note that the extended space is not compact.

A curve given by the equations

$$
\boldsymbol{\xi}_{j}=f_{j}(s),
$$$$
j=1, \cdots, N \text {, }
$$

with each $f_{j}$ continuous on a compact interval $[a, b]$, will be said to be a downward directed curve, with initial point that with ord value $b$, and endpoint that with ord value $a$. We shall allow $a=-\infty$ here. A boundary point of an open set will be said to be accessible from above if it is the endpoint of a downward directed curve which lies in the set, except for the endpoint. Note that this definition is applicable to the point $\infty$. A subset of an open set will be said to scan the open set if each point of the open set is the endpoint of a downward directed curve, lying in the open set, with initial point in the subset.

An open $N+1$ dimensional rectangle, with edges parallel to the coordinate axes, will be called a standard rectangle; the open infinite strip bounded by two horizontal hyperplanes will be called a standard strip. The closed upper bounding face of a standard rectangle will be called its upper boundary; the closure of the rest of the boundary will be called the lower boundary.

A function will be called parabolic if it is defined on a finite open set, if it has continuous second partial derivatives there, and if it satisfies the heat equation

$$
\frac{\partial u}{\partial s}=\frac{\partial^{2} u}{\partial \xi_{1}^{2}}+\cdots+\frac{\partial^{2} u}{\partial \xi_{N}^{2}}
$$

If $R$ is a standard rectangle, whose closure is in the domain of a parabolic function $u$, it is a classical fact that, if $z \in R$, or if $z$ is a boundary point of $R$ not on the lower boundary $C$, then $u(z)$ can be expressed in a simple way in terms of the values of $u$ on $C$, as follows. (For a treatment of parabolic functions on a standard rectangle, when $N=1$, see [5].) The value $u(z)$ is a weighted average of $u$ on $C$. The weighting is determined by a continuous density function (in $N$ dimensions) which vanishes at points above $z$, but is positive below $z$, except at the edges of the lower bounding face. The weight distribution on the boundary of $R$, determined in this way, will be called the parabolic measure relative to $R$, at $z$. The density determining this measure is easily expressed in terms of $\theta$ functions, and the regularity properties of these $\theta$ 
functions imply that parabolic functions have continuous partial derivatives of all orders.

Conversely, if $R$ is a standard rectangle, with lower boundary $C$, and if $f$ is any function defined on $C$, and integrable with respect to the parabolic measure relative to $R$, at each $z$ in $R$, then the integral of $f$ with respect to the parabolic measure at $z$, which we shall call the parabolic average of $f$ relative to $R$, at $z$, defines a function $u$, parabolic on $R$. If $f$ is Lebesgue integrable on the subset of $C$ in some neighborhood of the upper boundary of $R, u$ is even defined, by the above procedure, on the closure of $R$, less $C$. In particular, if $f$ is defined and continuous on $C$, and if $u$ is defined as $f$ on $C$, the function $u$ will now be defined and continuous on the closure of $R$.

A function $u$ will be called subparabolic if it is defined on a finite open set $D$, and if the following conditions are satisfied.

(i) $-\infty \leqq u<\infty$; $u$ is finite on a subset of $D$ which scans $D$.

(ii) $u$ is upper semicontinuous.

(iii) Let $R$ be a standard rectangle, with closure in $D$. If $v$ is defined and continuous on the closure of $R$, parabolic on $R, \geqq u$ on the lower boundary of $R$, then $u \leqq v$ on $R$.

In view of (i) and (ii), (iii) is equivalent to

(iii') Let $R$ be a standard rectangle, with closure in $D$. If $v(z)$ is defined as the parabolic average of $u$, relative to $R$, at $z$, then $u \leqq v$ on $R$.

If $z \in R$, and if $u(z)>-\infty$, (iii') implies that $u$ is Lebesgue integrable on sets of the lower boundary at $R$ at positive distance from the edges, and using (ii), it follows that $u$ is Lebesgue integrable on every compact subset of $D$ on a hyperplane which is parallel to a coordinate hyperplane, and that $u$ is Lebesgue integrable in $N+1$ dimensions on every compact subset of $D$. Thus the function $v$ of condition (iii') is necessarily finite, and is parabolic on $R$. It also follows that, in (iii), $u \leqq v$ on the closure of $R$.

A function will be called superparabolic if its negative is subparabolic. Then a function is parabolic if and only if it is both subparabolic and superparabolic. Since the study of subparabolic and superparabolic functions is in most respects similar to that of subharmonic and superharmonic functions, we shall only sketch the results we shall need, with no attempt at completeness.

Using (iii'), it follows readily that a continuous convex function of a parabolic function is subparabolic, and that a continuous convex monotone nondecreasing function of a subparabolic function is subparabolic.

EXAmple. As a simple example of a discontinuous subparabolic function, we note the function $u$, with domain the whole space, defined by

$$
u(z)=\left\{\begin{array}{rll}
-1 & \text { if } & \text { ord }(z)>0 \\
0 & \text { if } & \text { ord }(z) \leqq 0
\end{array}\right.
$$

TheOREm 1.1. A function $u$, defined on a finite open set, with continuous 
second partial derivatives, is subparabolic if and only if

$$
G u=\frac{\partial^{2} u}{\partial \xi_{1}^{2}}+\cdots+\frac{\partial^{2} u}{\partial \xi_{N}^{2}}-\frac{\partial u}{\partial s} \geqq 0 .
$$

In proving that (1.2) holds for subparabolic functions with continuous second partial derivatives, we can and shall suppose that the origin lies in the domain of $u$, and prove only that (1.2) is valid at that point. We give the proof for $N=1$, writing $\xi$ for $\xi_{1}$. Subtracting the proper multiples of the parabolic functions defined by the function values

$$
\xi, 2 s+\xi^{2}, 6 \xi s+\xi^{3}, s^{2}+\xi^{2} s,
$$

we can suppose that

$$
\frac{\partial u}{\partial \xi}=\frac{\partial u}{\partial s}=\frac{\partial^{2} u}{\partial \xi \partial s}=0, \quad \frac{\partial^{2} u}{\partial s^{2}}<0 \quad \text { if } \xi=s=0 .
$$

If $u$ does not have a local maximum at the origin, it follows that $\partial^{2} u / \partial \xi^{2} \geqq 0$ at that point. If $u$ does have a local maximum there, $u$ must be a constant in any sufficiently small standard rectangle with the origin on its upper boundary, so that $\partial^{2} u / \partial \xi^{2}=0$ at the origin. In either case, (1.2) is true there. Conversely, if $u$ satisfies (1.2), with $N=1$, we prove that $u$ is subparabolic. A particular case is the function defined by $u(\xi, s)=\xi^{2}$. This function is subparabolic, because it is a continuous convex function of the parabolic function defined by $u(\xi, s)=\xi$. Now let $u$ be given, satisfying (1.2), and consider the function $u_{c}$ defined by $u_{c}(\xi, s)=u(\xi, s)+c \xi^{2}$, where $c>0$. If we can prove that this function is subparabolic, for all $c>0$, it will follow by a trivial continuity argument that $u$ is also subparabolic. This function satisfies the inequality $G u_{c} \geqq 2 c$. Hence it is sufficient to prove that, if $G u>0$, then $u$ is subparabolic. Let $R$ be a standard rectangle whose closure lies in the domain of $u$, and let $v$ be parabolic on $R$, continuous on the closure of $R$, greater than or equal to $u$ on the lower boundary of $R$. We shall show that $u \leqq v$ on $R$, so that $u$ satisfies condition (iii). Let $z$ be a point of the closure of $R$ where $U=u-v$ has its maximum value on the closure. If $z$ is not on the lower boundary of $R$, then $\partial U / \partial \xi=0$, and $\partial U / \partial s \geqq 0$ at $z$, so that $\partial^{2} U / \partial \xi^{2}>0$ at $z$. Since the latter inequality is impossible at a maximum, $z$ must be on the lower boundary of $R$. Hence $U \leqq 0$ on $R$, as was to be proved.

THEOREM 1.2. If $u$ is subparabolic on the finite open set $D$, and if every limiting value of $u$ on the boundary, and when $|z| \rightarrow \infty$ if $D$ is unbounded, is $\leqq c$, then $u \leqq c$.

If the theorem is false, $u$ must have a maximum value $c_{1}>c$ at some point $z$ of $D$. But then it follows that $u$ is identically $c_{1}$ on any standard rectangle whose closure lies in $D$, and which has the point $z$ on its upper boundary. Then 
$u$ must be identically $c_{1}$ on every downward directed curve from $z$ which lies in $D$. This is obviously incompatible with the hypotheses of the lemma.

Note that, developing the argument of this proof, it follows that, if there is a point $z$ in $D$, with $u(z)=c, u$ will be identically $c$ in the subset of $D$ consisting of every point which is the endpoint of a downward directed curve in $D$ from $z$.

It is not obvious from our definition that subharmonicity is a local property, that is, that a function subparabolic in some neighborhood of each point of an open set is subparabolic on the set. However the following theorem states an even stronger result.

THEOREM 1.3. If a function $u$ satisfies the defining conditions (i), (ii) for subparabolic functions, then it is subparabolic if, for each $z$ in $D$, there is a neighborhood of $z$ such that the condition (iii), or the equivalent (iii'), is satisfied for every standard rectangle $R$ in this neighborhood, with $z$ a boundary point not on the lower boundary.

We shall call a function satisfying the conditions of this theorem subparabolic in the wide sense, and show that such a function is subparabolic. Obviously, if $u$ and $v$ have the same domain of definition, and are, respectively, subparabolic in the wide sense and parabolic, then the difference $u-v$ is subparabolic in the wide sense. Now suppose that $u$ is a function subparabolic on $D$, in the wide sense. We show that then condition (iii) for subparabolic functions is satisfied, so that $u$ is subparabolic. Let $R$ be a standard rectangle, with closure in $D$, and let $v$ be a function defined and continuous on the closure of $R$, parabolic on $R, \geqq u$ on the lower boundary of $R$. Then $u-v$ is subparabolic in the wide sense on $R$, and all limiting values of this difference on the lower boundary are $\leqq 0$. If the maximum value of this difference on the closure of $R$ is assumed on the lower boundary, then $u-v \leqq 0$ on $R$. If the maximum value $c$ is assumed at any other point $z_{0}$, and if $c>0$, then the local version of (iii') implies that $u-v$ has the value $c$ at all points of $R$ below $z_{0}$, contradicting the above stated properties of $u-v$ on the lower boundary of $R$. Thus $u-v \leqq 0$ on $R$, as was to be proved.

We shall frequently use the following easily proved fact that, if $\left\{u_{n}, n \geqq 1\right\}$ is a monotone nonincreasing sequence of subparabolic functions, with domain $D$, the limit $u$ is subparabolic on an open subset $D_{0}$ of $D$, and is $-\infty$ on $D-D_{0}$. Moreover, no point of $D-D_{0}$ is the endpoint of a downward directed curve lying in $D$, with initial point in $D_{0}$. In particular, $D_{0}$ may be empty, or may be $D$ itself, and the latter case must occur if it can be shown that $D_{0}$ scans $D$. If each $u_{n}$ is parabolic, $u$ is parabolic on $D_{0}$. Finally, if a sequence of non-negative subparabolic functions converges to 0 at a point $z$, it converges to 0 uniformly on every compact set whose points are endpoints of downward directed curves, lying in $D$, with initial point $\%$.

ExAmple. Define $\phi(z)$ by 


$$
\phi\left(\xi_{1}, \cdots, \xi_{N}, s\right)= \begin{cases}\frac{\exp \left(-\sum_{1}^{N} \xi_{j}^{2} / 4 s\right)}{\left(2(\pi s)^{1 / 2}\right)^{N}} & \text { if } s>0, \\ 0 & \text { if } s \leqq 0 .\end{cases}
$$

Then $\phi$ is lower semicontinuous. This function is parabolic except at the origin, because it satisfies the heat equation (1.1). The local average property of superparabolic functions for standard rectangles is trivially satisfied for the origin. Hence, by Theorem $1.3, \phi$ is superparabolic.

Let $\mu$ be a completely additive function of finite Borel sets of $N+1$ space finite on the bounded sets. Then we shall define the heat potential $u$ by

$$
u(z)=\int \phi(z-w) d \mu_{w}
$$

under certain restrictions on $\mu$. We first consider the case when $\mu$ is nonnegative and has value 0 on the complement of some compact set $A$. The integral (1.4) is the limit as $n \rightarrow \infty$ of the integral obtained by replacing $\phi$ in the integrand by $\phi_{n}=\operatorname{Min}[\phi, n]$. Then $\phi_{n}$ is continuous, and $u$ is a lower semicontinuous function, since the approximating integrals increase to $u$ and define continuous functions. The function $u$ is parabolic on the complement of $A$, since it satisfies the heat equation there, and obviously $u$ vanishes below $A$. Moreover, $u$ is superparabolic, as is shown by the following argument. The function $\phi$ is superparabolic. Hence $\phi_{n}$, as the negative of a convex monotone nondecreasing function of $-\phi$, is superparabolic, so the weighted average $u_{n}$, defined by (1.4) with $\phi$ replaced by $\phi_{n}$ is superparabolic. Finally, using the remarks made above on monotone sequences of subparabolic functions, the limit $u$ of the monotone sequence $\left\{u_{n}, n \geqq 1\right\}$ is superparabolic.

If $\mu$ is supposed non-negative, but not necessarily confined to a compact set, the corresponding heat potential (1.4), under a restriction on $\mu$ to be given below, is defined by

$$
u(z)=\lim _{n \rightarrow \infty} \int_{A_{n}} \phi(z-w) d \mu_{w},
$$

where $A_{n}$ is finite and compact, $A_{1} \subset A_{2} \subset \cdots$, and $\cup_{1}^{\infty} A_{n}$ is finite $N+1$ space. The limit is obviously independent of the choice of these sets. According to what we have just shown, the $n$th integral in $\left(1.4^{\prime}\right)$ defines a superparabolic function which does not decrease when $n$ increases. Hence $u$ must have one of the following three characterizations:

(a) $u$ is identically $+\infty$;

(b) there is a horizontal hyperplane such that $u$ is identically $+\infty$ above the hyperplane, and superparabolic below it;

(c) $u$ is superparabolic. 
We shall always restrict $\mu$ by supposing that we are in case (c), that is, we only define the potential in this case. The potential will then be defined for example, if $\mu$ is finite valued and vanishes on sets above some horizontal hyperplane.

If $\mu$ is supposed nonpositive, the corresponding heat potential is defined as the negative of that defined by $-\mu$. In the general case, $\mu$ can be written as the sum of its positive and negative variations, and $u$ is defined as the sum of the corresponding potentials, assuming that both are defined. The set function $\mu$ will be called a distribution of heat sources, positive, negative, or mixed, as the case may be.

Let $u$ be parabolic on a standard strip bounded by the hyperplanes with ordinate values $a, b, a<b$, and let $z:\left(\xi, \cdots, \xi_{N}, s\right)$ be a point of the strip. Then $u(z)$ is equal to the parabolic average of $u$ over the lower boundary of a standard rectangle $R_{1}$ whose closure lies in the given standard strip $R$, and which has $z$ on its upper boundary. If $a<t<s<b$, we can take $t$ as the ordinate value of the lower bounding face of $R_{1}$, and then let the other faces of the lower boundary recede indefinitely. In this way, if the contribution to the parabolic average of $u$ from the receding faces goes to 0 , one can obtain a simple expression for $u(z)$,

$$
u(z)=\frac{\int_{-\infty}^{\infty} \cdots \int_{-\infty}^{\infty} u\left(\eta_{1}, \cdots, \eta_{N}, t\right) \exp \left(\frac{-\sum_{1}^{N}\left(\xi_{j}-\eta_{j}\right)^{2}}{4(s-t)}\right) d \eta_{1} \cdots d \eta_{N}}{\left(2 \pi^{1 / 2}(s-t)^{1 / 2}\right)^{N}} .
$$

This method leads to (1.5) with inequality ( $\leqq$ ), if $u$ is subparabolic. This derivation can be carried through if, for some positive constant $c$,

$$
|u(z)| \leqq c \exp \left(\sum_{1}^{N} \xi_{j}^{2} / 4 N(b-a)\right) \quad \text { if } a<s<b,|z| \geqq c,
$$

in the parabolic case, if

$$
u(z) \leqq c \exp \left(\sum_{1}^{N} \xi_{j}^{2} / 4 N(b-a)\right) \quad \text { if } a<s<b,|z| \geqq c,
$$

in the subparabolic case. In deriving (1.5), or the corresponding inequality in the subparabolic case, one can profitably apply the probability interpretation of parabolic averages to be described in $\$ 2$, together with the known evaluation of the probability distribution of the maximum of a one dimensional Brownian motion sample function over a parameter interval. The details of the simple calculations will be omitted, in view of the fact that the justification becomes trivial in the only case essential to the work of this paper, that in which $u(z) \rightarrow 0$ when $|z| \rightarrow \infty$. Finally, we remark that, if $N=1$, and if $u$ is parabolic and non-negative, the validity of the Poisson integral formula (1.5) is a trivial consequence of a theorem of Widder [7]. 
If $u$ is subparabolic on an open set $D$, properties (ii) and (iii') of the definition of parabolicity taken together imply that, if $z \in D$,

$$
u(z)=\underset{w \rightarrow z}{\limsup } u(w) \quad[\operatorname{ord}(w)<\operatorname{ord}(z)] .
$$

Finally, the same properties imply that, if $R$ is a standard rectangle with $z$ a boundary point not on the lower boundary, then the parabolic average of $u$ on the lower boundary of $R$, at $z$, converges to $u(z)$ when $R$ shrinks to the point $z$. Moreover, if $R_{1}, R_{2}$ are two such rectangles, whose closures are in $D$, and if $R_{1} \subset R_{2}$, the parabolic average of $u$ at $z$ on the lower boundary of $R_{1}$ is less than or equal to that on the lower boundary of $R_{\mathbf{2}}$. The proof will be omitted, since it is the exact analogue of the proof of the corresponding theorem for subharmonic functions. This result will be considerably generalized below.

Let $R_{a}(z)$ be the standard rectangle whose edges parallel to the $s$ axis have length $a^{2}$, whose other edges have length $a$, and which has $z$ at the center of symmetry of its upper bounding face. Then, if $u$ is subparabolic on an open set $D$, and if $z \in D, u(z)$ is less than or equal to the parabolic average $v_{a}(z)$ of $u$ on the lower boundary of $R_{a}(z)$, at $z$, for sufficiently small $a$. According to the preceding paragraph, this parabolic average does not decrease when $a$ increases. Conversely, this property, which we shall call (iii"), can be used to replace the property (iii') in the definition of superparabolicity. This assertion is a slight strengthening of Theorem 1.3, and the proof of that theorem, with the obvious changes, proves the assertion.

The parabolic measure on the lower boundary of $R_{1}(z)$, at $z$, is determined by the density function $f$ say, where for simplicity we take $z$ as the origin. Then it is easy to see that the corresponding density value for $R_{a}(z)$ is given by

$$
a^{-N} f\left(\xi_{1} / a, \cdots, \xi_{N} / a, s / a^{2}\right)
$$

on the horizontal face of the lower boundary, and by

$$
a^{-N-1} f\left(\xi_{1} / a, \cdots, \xi_{N} / a, s / a^{2}\right)
$$

on the faces parallel to the $s$ axis. If the inequality $u(z) \leqq v_{a}(z)$ is integrated with respect to $a$, from 0 to $\delta$, with weighting function $(N+2) a^{N+1} / \delta^{N+2}$, we then obtain an inequality $u(z) \leqq u_{\delta}(z)$, where $u_{\delta}$ is an average of $u$ over $R_{a}(z)$, with a continuous weighting function. Obviously this inequality, for sufficiently small $\delta$, can be used to replace the condition (iii') for subparabolicity. Since $u_{\delta}$ is an average over an $N+1$ dimensional set, $u_{\delta}$ is a continuous function of $z$ on the subset of $D$ at distance less than $(a / 2)(4 N+1)^{1 / 2}$ from the boundary of $D$. Finally, $u_{\delta}$ is subparabolic on its domain, because it is readily checked that the property (iii") is satisfied. Thus, just as in the case of subharmonic functions, we have found a decreasing sequence $\left\{u_{1 / n}+1 / n, n \geqq 1\right\}$ of continuous subparabolic functions converging to the given subparabolic function $u$. Iterating this procedure, we can find a decreasing sequence of 
subparabolic functions, with any specified number of continuous partial derivatives, converging to $u$.

We conclude this section with some remarks on the relations of the definitions we have given to the corresponding definitions in the study of Laplace's equation. If $u$ is defined on an open set $D$ which contains with every point the whole line parallel to the $s$ axis through that point, and if $u$ is a function of the space variables $\left(\xi_{1}, \cdots, \xi_{N}\right)$ only, then $u$ is subparabolic [parabolic] if and only if the function $v$ of $\left(\xi_{1}, \cdots, \xi_{N}\right)$ it defines is subharmonic [harmonic] on its domain in $N$ space. If $u$ has continuous second partial derivatives, the proof follows from the fact that the differential inequalities or equations in the one case become exactly those relevant to the other case. If these derivatives do not exist, the functions are approximated as described above by functions of the same type for which the derivatives exist. Note that, if $u$ is a function of the space variables only, the approximating functions we have found have this same property.

If $\mu$ is a distribution of heat sources, generating a potential $u$, and if $\mu$ is invariant under translations in the direction of the $s$ axis, $\mu$ can be written as the direct product of a completely additive function $\nu$ of Borel sets in $N$ dimensions, that is, of a distribution on the space of the $\xi_{j}$ 's, and Lebesgue measure on the $s$ axis. If $N \geqq 3$, the corresponding potential will then be a function $v$ of the $\xi_{j}$ 's, and in fact will be the ordinary Newtonian potential of $c_{N} \nu$, where

$$
\begin{aligned}
c_{N} & =r^{N-2} \int_{-\infty}^{s} \frac{\exp \left(-r^{2} / 4(s-t)\right)}{\left(2 \pi^{1 / 2}\right)^{N}(s-t)^{N / 2}} d t \\
& =\int_{0}^{\infty} \frac{e^{-\alpha^{2} \alpha^{N-3}}}{2 \pi^{N / 2}} d \alpha .
\end{aligned}
$$$$
\left(r^{2}=\sum_{1}^{N}\left(\xi_{j}-\eta_{j}\right)^{2}\right)
$$

Thus, neglecting a constant factor, the heat potential reduces to the ordinary Newtonian potential, in this special case. Conversely, any Newtonian potential in $N \geqq 3$ dimensions defines a heat potential with the heat source distribution of the special type described above. The case $N=2$ is of course exceptional, and will be treated when Green potentials, that is, potentials relative to arbitrary open sets, are treated.

2. Brownian trajectories. Let $\{x(t), 0 \leqq t<\infty\}$ be an $N$ dimensional Brownian motion process. More specifically, the following is supposed. There is a space $\Omega$ of points $\omega$. Functions on $\Omega$ will be denoted by $x, y$, and so on, their values at $\omega$ by $x(\omega), y(\omega)$, and so on. A probability measure $P$ is defined on a Borel field of sets of $\Omega$. For each $t \geqq 0, x(t)$ is an $N$ dimensional measurable vector function on $\Omega$, with $x(0)=0$. The $x(t)$ process is Gaussian; its $N$ component processes are mutually independent, and, if $\{y(t), 0 \leqq t<\infty\}$ is any one of these component processes,

$$
E\{y(t)\}=0, \quad E\left\{|y(t)-y(s)|^{2}\right\}=|t-s| / 2 .
$$


Finally, it is supposed that almost all sample functions of the $y(t)$ process, that is, the functions of $t$ obtained by fixing $\omega$, for almost all $\omega$, are continuous.

If $0 \leqq t_{0}<\cdots<t_{n}$, if $A_{1}, \cdots, A_{n}$ are $N$ dimensional Borel sets, and if $\xi_{0}$ is a point of $N$ space, it is clear that

$$
P\left\{x\left(t_{j}, \omega\right)+\xi_{0} \in A_{j}, j=1, \cdots, n\right\}
$$

defines a Baire function of $\xi_{0}$. The probabilities and expectations relating to Brownian motions that we discuss in this paper are at worst limits of linear combinations of probabilities of the above form, and therefore define Baire functions of $\xi_{0}$. This fact will be used without special comment below.

From now on, we deal with $N+1$ space as in $\S 1$. Let $z_{0}:\left(\xi_{0} \cdot s_{0}\right)$ be a finite point of this space, where $\xi_{0}$ represents the first $N$ coordinates of $z_{0}$. When we describe a stochastic process as a Brownian trajectory process with initial point $z_{0}$, we shall always mean a stochastic process $\{z(t), 0 \leqq t \leqq \infty\}$, where $z(t)$ is a random variable with values points of extended $N+1$ space, given by

$$
z(t)=\left(\xi_{0}+x(t), s_{0}-t\right),
$$

if $t<\infty$,

and $z(\infty)$ is identically the point $\infty$. Here the $x(t)$ process is a Brownian motion process in $N$ dimensions, as specified above. The Brownian trajectories, that is, the sample curves of the Brownian trajectory process, are then Brownian motion sample functions graphed vertically, with initial point $z_{0}$, and with the point $\infty$ adjoined as endpoint of each trajectory.

Given a Brownian trajectory process, with initial point $z$, and a closed set $C$ of extended $N+1$ space, we shall denote by $Z(z, C)$ the point where a trajectory first meets $C$. The function $Z(z, C)$ is a point-valued random variable. In simple cases, the distribution of this random variable has been calculated, in a different setting. For example, let $N=1$, and let $C$ be the lower boundary of a standard rectangle $R$. Let the Brownian trajectory process be given by (2.1), and let $R$ have the diagonally opposite vertices $\left(\xi_{0}-a, s_{0}\right)$, $\left(\xi_{0}+b, s_{0}-t\right)$, where $a, b$ are positive numbers. Then the probability that $Z\left(z_{0}, C\right)$ lies in a set of the lower bounding segment of $C$ is the probability that the value of $z(t)$ lies in this set, and that

$$
a<x(s, \omega)<b
$$$$
\text { if } 0 \leqq s \leqq t .
$$

The remaining probability relations involving $Z\left(z_{0}, C\right)$ are expressed similarly in terms of known distributions connected with Brownian motion processes. These distributions were first obtained by Bachelier, using the reflection principle of André, and the corresponding distributions for $N>1$ are then easily obtained also. Since the André reflection principle corresponds exactly to that used in finding solutions of the heat equation, it follows that the distribution of $Z\left(z_{0}, C\right)$ is, in this case, precisely the parabolic measure on $C$, at $z_{0}$. In other words, if $u$ is parabolic on a domain containing the closure of the standard rectangle $R$, or, more generally, if $u$ is merely continuous on the closure of $R$ and parabolic on $R$, and if $z \in R$, then 


$$
u(z)=E\{u[Z(z, C)\rfloor\},
$$

where $C$ is the lower boundary of $R$. Conversely, we have noted above, in different language, that, if $f$ is a function defined and continuous on $C$, $E\{f[Z(z, C)]\}$ defines a function on the closure of $R$ which is continuous, parabolic on $R$, and identical with $f$ on $C$. If $D$ is any finite open set, with boundary $S$, and if $z \in D$, the distribution of $Z(z, S)$ defines a measure of Borel subsets of $S$ which we shall call, when completed in the usual way, the parabolic measure on $S$, relative to $D$, at $z$. The integral of a function $f$ with respect to this measure will be called the parabolic average of $f$, relative to $D$, at $z$. If the point $\infty$ has positive parabolic measure $p(z)$ relative to $D$ at $z$, this point contributes $f(\infty) p(z)$ to the parabolic average of $f$. Thus the value of $f$ at $\infty$, if finite, is irrelevant to the integrability of $f$.

The parabolic measure relative to $D$ at $z$ determines an $L_{1}$ class of functions on the boundary, which we shall denote by $L(z, D)$. The intersection of all these classes, for $z$ varying on $D$, will be denoted by $L(D)$. In particular, if $D$ is a standard rectangle, we have remarked that the parabolic measure on $S$ is given by a continuous density function on the part of $S$ below $z$. In this case, the class $L(z, D)$ does not change when $z$ moves horizontally, but increases when ord $(z)$ decreases. This illustrates one way in which parabolic measure is less simple than harmonic measure.

Theorem 2.1. Let $D$ be a finite open set, with boundary $C$, and suppose that $f \in L(z, D)$, for each $z$ in some open subset $D_{0}$ of $D$. Then the equation

$$
u(z)=E\{f[Z(z, C)]\}
$$

defines a function parabolic on $D_{0}$.

We have already remarked that any expectation of the type defining $u$ here defines a Baire function. If $R$ is a standard rectangle, whose closure is in $D, u(z)$ is the parabolic average of $u$ on the lower boundary $S$ of $R$, relative to $R$ at $z$. In fact this statement is simply an expression of the fact that, when a Brownian trajectory from $z$ first meets $S$, the meeting point is $Z(z, S)$, and the distribution of $Z(z, C)$ can be obtained either by continuing this trajectory or by starting a new one from $Z(z, S)$, independent of the old one. This is an application of the André reflection principle, and is thus another application of the principle used to obtain the evaluation of the parabolic measure on the boundary of a standard rectangle, which forms the basis of this paper. Formally, we can write, if $z \in R$, using the standard theorems on iteration of conditional expectations

$$
u(z)=E\left\{f_{1}[Z(z, C)]\right\}
$$

where $f_{1}$ is defined on $C$ by

$$
f_{1}\left(z_{1}\right)=E\left\{f\left[Z\left(z_{1}, S\right) \mid\right\}=u\left(z_{1}\right) .\right.
$$


that is, $u$ is the parabolic average of $f_{1}$ on $S$. We have already remarked that this average property characterizes parabolic functions.

If $f$ is a Baire function defined on the boundary $C$ of an open set $D$, Theorem 2.1 can be used to give insight into the set of values of $z$ in $D$ for which $f \in L(z, D)$. We can assume that $f \geqq 0$ (or replace $f$ by $|f|$ ). Let

$$
f_{n}=\operatorname{Min}[f, n] \text {, }
$$

and define $u_{n}$ on $D$ as the parabolic average of $f_{n}$ relative to $D$. Then the sequence $\left\{u_{n}, n \geqq 1\right\}$ is monotone nondecreasing, with limit $u$, and we have seen that there is a (possibly empty) open subset $D_{0}$ of $D$ on which $u$ is finite and parabolic, and that $u(z)=+\infty$ on $D-D_{0}$. The set $D_{0}$ consists of those values of $z$ with $f \in L(z, D)$, and no point of $D-D_{0}$ is the endpoint of a downward directed curve, lying in $D$, with initial point in $D_{0}$.

Example. If $D$ is a finite open set, and if $A$ is an $F_{\sigma}$ set, contained in $D$, define $u_{A, D}$ at each point $z$ in $D$ as the probability that a Brownian trajectory from $z$ passes through a point of $A$ before it meets the boundary of $D$. This function is the analogue, or rather the generalization, of the classical equilibrium potential of the usual potential theory. In fact, suppose that $A$ and $D$ are unions of aggregates of lines parallel to the $s$ axis, that is, $A[D]$ is the direct product of an $F_{\sigma}$ [open] set $A_{0}\left[D_{0}\right]$ in $N$ dimensions and the $s$ axis. Then $u_{A, D}$ becomes a function of the $N$ space variables, defined on $D_{0}$, and is the classical equilibrium potential relative to the boundary of $D_{0}$, according to [2]. In the classical theory, $D_{0}$ is usually taken as $N$ space or a sphere if $N>2$, and is taken as a disc if $N=2$. For our purposes, it will be unnecessary to differentiate between the various values of $N$. Even the case $N=1$, which is no longer a trivial special case, will not have to be distinguished from the others. If $D$ is the whole finite $N+1$ space, $u_{A, D}$ becomes the probability that a Brownian trajectory from $z$ ever passes through a point of $A$. With this choice of $D$, we shall write $u_{A}$ instead of $u_{A, D}$.

THEOREM 2.2. The function $u_{A, D}$ has the following properties.

(i) $u_{A, D}$ is superparabolic.

(ii) $u_{A, D}$ is parabolic on every open subset of $D-A$.

(iii) $0 \leqq u_{A, D} \leqq 1$; there is equality on the right at the interior points of $A$, and on the left at points below $A$.

The function $u_{A, D}$ obviously has property (iii). To treat the other properties, it will be convenient to suppose first that $A$ is closed in the unextended topology, and we shall do so. Let $f$ be the function defined as 1 on $A$ and 0 on the boundary of $D$. Then, if $z \in D-A$, and if $S$ is the boundary of $D-A$,

$$
u_{A, D}(z)=E\{f[Z(z, S)]\} .
$$

Hence, according to Theorem 2.1, (ii) is true. To prove that $u_{A, D}$ is lower semicontinuous, let $z$ be an arbitrary point of $D$, and let $A_{r}$ be $A$ less the interior of a sphere with center $z$, radius $r$. Then 


$$
u_{A, D}(w)=\lim _{r \rightarrow 0} u_{A_{r}, D}(w)
$$

on $D$. Since $u_{A-, D}$ is continuous at $z$, by (ii), $u_{A, D}$ must be lower semicontinuous there. To prove that $u_{A, D}$ has the average property of superparabolic functions at $z \in D$, we need only remark that $u_{A, D}(z)$ is greater than or equal to the probability that a Brownian trajectory from $z$ will meet $A$ before the boundary of $D$, after first crossing the boundary of a standard rectangle which contains $z$ and whose closure lies in $D$. This statement is precisely the average property. Thus $u_{A, D}$ is superparabolic. Finally, if $A$ is no longer supposed closed in the unextended topology but is an arbitrary $F_{\sigma}$ set, we can write $A=\mathrm{U}_{1}^{\infty} B_{n}$, where $B_{n}$ is closed in the unextended topology and does not decrease when $n$ increases. Then

$$
u_{A, D}=\lim _{n \rightarrow \infty} u_{B_{n}, D}
$$

The fact that (i) and (ii) are true for $u_{B_{n}, D}$ implies their truth for $u_{A, D}$.

3. The Dirichlet problem for the heat equation. Let $D$ be any open set of $N+1$ space, with boundary $C$. The Dirichlet (first boundary value) problem for the heat equation is the following, at least in the classical form. Let $f$ be a function defined and continuous on $C$. A function $u$ is to be found, continuous on $D \cup C$, equal to $f$ on $C$, parabolic on $D$. More generally, as usual in modern discussions of the Dirichlet problem, it will not be supposed that $f$ is necessarily continuous, and $u$ will not be required to have the assigned boundary value at each boundary point. However, it is not to be expected that even the problem as originally stated has a solution unless the boundary has special properties. That is, the boundary points will have to be classified as regular and irregular, in the now standard senses.

The Dirichlet problem for the heat equation has been treated by many authors, but, for various reasons, has not been described in these terms. For $N=1$, the set $D$ has usually been the domain bounded from above and below by horizontal line segments, and laterally by downward directed curves. Now it is clear that the upper bounding segment of such a domain plays a special role. In fact the prescription of a continuous boundary function on the boundary less the points of the upper segment is sufficient to determine the desired solution $u$, so that $f$ cannot be prescribed arbitrarily on the upper segment. This fact, whose proper interpretation is simply that the inner points of the upper bounding segment are irregular, has obscured the fact that the usual notions in the Dirichlet problem are applicable, because the upper bounding segment seems at first to be too large a class of exceptional points. One thinks of the class of exceptional boundary points of a boundary in a Dirichlet problem as a small class, and a line segment does not seem small enough in two dimensions. However this is an unjustifiable prejudice, and we shall see that, in a perfectly reasonable sense, the class of irregular boundary points of a domain $D$ of the type under discussion has zero measure. The sense in which 
this is true for the most general domain is still in part an open question.

Let $D$ be a finite open set in $N+1$ space, with boundary $C$. Let $f$ be a function defined on $C$, and suppose that $f \in L(D)$. For example, any bounded Baire function satisfies this condition. Then the parabolic average of $f$ rela. tive to $D$, that is, the function with domain $D$ defined by the equation

$$
u(z)=E\{f[Z(z, C)]\},
$$

will be called the Dirichlet solution corresponding to the boundary function $f$. According to Theorem 2.1, $u$ is parabolic. Somewhat more generally, it will be convenient to define an "extended Dirichlet solution." In this extension we assume that either Min $[f, 0]$ or $\operatorname{Max}[f, 0]$ is a member of the class $L(D)$, and define $u$ as above. Then we have seen that $f \in L(z, D)$ on a certain open set $D_{0}$, which may be empty, so that $u$ is parabolic on $D_{0}$, and $u(z)=+\infty$ or $u(z)=-\infty$ on $D-D_{0}$, depending on which of the two above hypotheses is satisfied. No point of $D-D_{0}$ is the endpoint of a downward directed curve lying in $D$, with initial point in $D_{0}$.

Our definition of the Dirichlet solution will be justified by the theorems in this and later sections. If $D$ is a union of lines parallel to the $s$ axis, $\infty$ is a boundary point. We shall suppose that the boundary set containing only this one point has parabolic measure 0 on $D$, so that we can neglect this point in discussing Dirichlet solutions. Suppose that $f$ does not depend on $s$. Then $f$ and $u$ reduce to functions of the $N$ space variables, and from this point of view $u$ becomes the solution of the Dirichlet problem for Laplace's equation in $N$ dimensions, with the boundary function determined by $f$. This is precisely the form given to the Dirichlet solution for Laplace's equation in [2]. The restriction on the role of the point $\infty$ means that almost every Brownian motion in $N$ dimensions starting from a point of the $N$ dimensional projection of $D$ meets the boundary.

As is customary, we define a boundary point as regular if, whenever the boundary function $f$ is bounded, and continuous at the point in question, $u$ has the value of $f$ at that point as boundary limit. Clearly, a finite point $z_{0}$ is regular if and only if the distribution of $Z(z, C)$, that is, the parabolic measure relative to $D$ at $z$, becomes concentrated at $z_{0}$ when $z \rightarrow z_{0}$. More precisely, the finite boundary point $z_{0}$ is regular if and only if

$$
p \lim _{z \rightarrow z_{0}, z \in D} Z(z, C)=z_{0},
$$

where " $p$ lim" means limit in probability. The classical argument shows that, if $f$ is bounded from above, and if $z_{0}$ is a finite regular boundary point, then

$$
\lim _{z \rightarrow z_{0}, z \in D} u(z) \leqq \lim _{z \rightarrow z_{0}, z \in C} \sup _{z} f(z) \text {. }
$$

The corresponding result holds if $f$ is bounded from below. It is a trivial fact that the point $\infty$ is a regular boundary point of every finite open set which 
is not bounded from below. An essential difference between $\infty$ and other boundary points is that $\infty$ may have positive parabolic measure, whereas no single finite boundary point can have positive parabolic measure at any point of $D$.

We shall now proceed to derive a simple condition for regularity of a finite boundary point, containing as a special case a corresponding one (see [2]) relevant to Laplace's equation. Consider the trajectories of a Brownian trajectory process, with initial point $z_{0}$, a finite boundary point of the finite open set $D$. Then it is clear from the usual zero-one law reasoning, applied to a Brownian motion process, that either almost every such trajectory meets the complement of $D$ less the point $z_{0}$ arbitrarily soon, or else almost every trajectory lies in $D$ for an initial open parameter interval, of length depending on the trajectory. These two possibilities characterize the regularity or irregularity of the boundary point, according to the following theorem. This theorem was proved by Fortet [4] for $N=1$, for a rather special class of domains, but for a somewhat more general basic parabolic equation than the heat equation.

THEOREM 3.1. The boundary point is regular in the first case, irregular in the second.

If a trajectory from $z_{0}$ has any point in $D$ near $z_{0}$, the trajectory will have probability nearly 1 of returning to meet the boundary $C$ near the starting point, if $z_{0}$ is a regular boundary point. But then almost every trajectory from $z_{0}$ will either not enter $D$ at all near $z_{0}$, or, if it does, will meet the boundary again shortly thereafter. Hence, in the regular case, almost no trajectory from $z_{0}$ lies in $D$ for an initial open parameter interval. Conversely, suppose that $z_{0}$ is irregular, so that there is a positive number $\delta$ with the property that there is probability $>\delta$ that trajectories from certain points in $D$ arbitrarily close to $z_{0}$ reach $C$ at the earliest after time $\delta$. We can and shall now assume that $D$ contains all points $z$ with

$$
\operatorname{ord}(z)<\operatorname{ord}\left(z_{0}\right)-\delta \text {. }
$$

Then $u_{C}\left(z_{0}\right)<1-\delta$ for points in $D$ arbitrarily near $z_{0}$. Since $u_{C}$ is lower semicontinuous, $u_{C}\left(z_{0}\right) \leqq 1-\delta$. Thus there is probability at least $\delta$ that a Brownian trajectory from $z_{0}$ lies in $D$ for an initial open parameter interval of length $\geqq \delta$, as was to be proved.

According to Theorem 3.1, the regularity of a boundary point is not only a local property of the boundary near the point, but, more than that, the regularity of $z_{0}$ depends only on the character of $D$ in the part of an arbitrarily small neighborhood of $z_{0}$ below $z_{0}$. It follows that $z_{0}$ is regular if and only if (3.1) is true with $z \rightarrow z_{0}$ from below.

Since the sample functions of one dimensional Brownian motion processes have infinite upper and lower derivatives at any specified parameter point, 
with probability 1 , the irregular points of simple boundaries are easily identified. For example, let $N=1$, and let $D$ be an open disc, less the points of a horizontal radius. Then it is clear from the above property of Brownian motion sample functions that all perimeter points of $C$ are regular except possibly the one with maximum ord value. An application of the iterated logarithm law yields the regularity of this point also. On the other hand, it is also clear that the points of the bounding radius not on the perimeter are irregular. If the bounding radius is not horizontal, its points are regular boundary points also. Note that, if the two sides of the bounding radius less the center are counted separately (prime end theory), with the obvious corresponding definition of regularity of a boundary point, then, in this example, if the bounding radius is horizontal, the points of its upper side (less the center) are regular, those on the lower side (less the point at the perimeter) are irregular. In this example, corresponding to the fact that the lower side of the radius less one endpoint is irregular, is the fact that the parabolic measure of the lower side relative to $D$ vanishes identically.

As an extreme example, consider the case in which $N=1$ and in which $D$ is a standard rectangle. Then only the points of the lower boundary are regular. Let $f$ be a boundary function which is non-negative, continuous on the lower boundary except at the two points $z_{1}, z_{2}$ on the upper boundary, where $f$ becomes infinite. The function $f$ can be defined arbitrarily or not at all on the upper boundary. Then $f \in L(D)$, and the corresponding Dirichlet solution $u$ takes on the assigned boundary value at every point of the lower boundary, including $+\infty$ at $z_{1}, z_{2}$. If $f$ has a finite integral on the lower boundary, $u$ takes on a boundary limit value at each point of the upper boundary, finite except at $z_{1}, z_{2}$, defining a continuous boundary function (in the obvious extended sense at $z_{1}, z_{2}$ ). If $f$ has an infinite integral on the lower boundary, $u$ has the boundary function $+\infty$ on the upper boundary.

As a further example, suppose that $N=1$ and that the boundary $C$ of $D$ contains an arc defined by the equation

$$
\xi=\psi(s), \quad a \leqq s \leqq b,
$$

where $\psi$ is continuous, and that each point of the arc with $a<s<b$ is at positive distance from the boundary less the arc. Let $z_{0}:\left[\psi\left(s_{0}\right), s_{0}\right]$ be a point of this arc with $a<s_{0}<b$, and suppose for definiteness that $D$ contains all points near this arc, to the left of the arc, none to the right. Then the condition of Theorem 3.1 for irregularity of $z_{0}$ is that, if $\{x(t), 0 \leqq t<\infty\}$ is a Brownian motion process in one dimension, as defined in $\$ 2$,

$$
x(t)<\psi\left(s_{0}-t\right)-\psi\left(s_{0}\right)
$$

for sufficiently small $t$, with probability 1 . Conditions for this to be true are well known, under various restrictions on $\psi$. For example if $\theta(t)$ is the right side of the preceding inequality, and if $\theta$ is a monotone nondecreasing function of 
$t$, for small positive $t$, the above condition is satisfied, according to Kolmogorov, if and only if

$$
\int_{0+} \theta(t) e^{-\theta(t)^{2} / 4 t t^{-3 / 2}} d t<\infty .
$$

From an analytic point of view, this analysis of the regularity of the points of such a bounding curve goes back to Petrowsky [6].

The following theorem is standard in this type of discussion. Since we shall use it, and since its proof has one slightly unorthodox touch, we state and prove it in detail, in so far as the details are not obvious.

THEOREM 3.2. Let $u$ be a function subparabolic on a finite open set containing the closure, assumed compact, of an open set $D$. Suppose that every point of the boundary $C$ of $D$ is regular, and define $f$ on $C$ as $u$. Then $f \in L(D)$, and, if $v$ is the Dirichlet solution on $D$ determined by the boundary function $f, u \leqq v$ on $D$. Moreover, the function $u_{1}$ defined as $v$ on $D$ and $u$ otherwise is subparabolic on the domain of $u$.

It follows readily from results to be obtained below that, except for the last assertion, this theorem remains true even if $C$ does not consist solely of regular points. Since $u$ is upper semicontinuous, $f$ is also, and is therefore bounded from above. Let $\left\{f_{n}, n \geqq 1\right\}$ be a monotone nonincreasing sequence of continuous functions on $C$, with $\operatorname{limit} f$, and let $v_{n}$ be the Dirichlet solution on $D$ determined by the boundary function $f_{n}$. Then, applying Theorem 1.2, and using the fact that the points of $C$ are regular, we find that $u \leqq v_{n}$ on $D$. Thus, if $v$ is the extended Dirichlet solution on $D$ determined by the boundary function $f$, we find that $u \leqq v$ on $D$. Hence $v$ is finite on a dense subset of $D$, so that $v$ is parabolic, $f \in L(D)$, and $v$ is an ordinary Dirichlet solution. The fact that $u_{1}$ is subparabolic is trivial to verify.

We can now prove the following theorem, which will be used to show that reasonably well-behaved parabolic functions are in fact Dirichlet solutions. It will be generalized considerably below.

THEOREM 3.3. Let $u$ be subparabolic, and bounded from above, on the finite open set $D$. Define $f$ at each finite or infinite boundary point $z_{0}$ of $D$ which is accessible from above by the equation

$$
f\left(z_{0}\right)=\lim _{z \rightarrow z_{0}} u(z) .
$$

Then $f \in L(D)$ and $u$ is less than or equal to the Dirichlet solution on $D$ determined by the boundary function $f$.

Since the parabolic measure relative to $D$ of the boundary set where $f$ is not defined vanishes on $D$, and since $f$ is bounded from above, $f$ must determine either a Dirichlet solution, or at least an extended one. Let $D_{n}$, with 
boundary $C_{n}$, be a bounded open set, all of whose boundary points are regular, whose closure lies in $D$, and such that

$$
D_{1} \subset D_{2} \subset \cdots, \quad \bigcup_{1}^{\infty} D_{n}=D .
$$

Let $f_{n}$ be the function with domain $C_{n}$, where it coincides with $u$. Then, according to the preceding theorem, $f \in L\left(D_{n}\right)$, and, if $v_{n}$ is the Dirichlet solution on $D_{n}$ determined by the boundary function $f_{n}$,

$$
v_{n}(z)=E\left\{f_{n}\left[Z\left(z, C_{n}\right)\right]\right\},
$$$$
z \in D_{n},
$$

it follows that $u \leqq v_{n}$ in $D_{n}$. Now fix $z$, and consider $Z\left(z, C_{n}\right)$ as $n$ varies. We can and shall assume that $Z\left(z, C_{n}\right)$ is defined, for fixed $z$, using the same Brownian motion process for all $n$. If this is done, and if $C$ is the boundary of $D$, it is clear that $Z\left(z, C_{n}\right) \rightarrow Z(z, C)$ with probability 1 , so that

$$
\limsup _{n \rightarrow \infty} f_{n}\left[Z\left(z, C_{n}\right)\right] \leqq f[Z(z, C)]
$$

with probability 1 . Since $u$ is bounded from above, the sequence of functions involved has this same property, and it follows that

$$
u(z) \leqq \limsup _{n \rightarrow \infty} v_{n}(z) \leqq E\{f[Z(z, C)]\} \quad \text { if } z \in D .
$$

Since $u$ is subparabolic, it is finite on a dense set. Hence the preceding inequality implies that $f \in L(D)$, and thereby the truth of the theorem.

It is clear that this result could be strengthened as follows. Instead of the stated definition of $f$ it need only be supposed that $f$ is a function defined on $C$, is bounded from above, is a Baire function, and has the property that, excluding perhaps a subset of $C$ which has parabolic measure 0 relative to $D$, identically on $D, f\left(z_{0}\right)$, for $z_{0} \in C$, is at least equal to the limit superior of $u$ at $z_{0}$, taken along any downward directed curve lying in $D$ except for the endpoint $z_{0}$. The hypothesis that $u$ be bounded from above can also be weakened. It will be shown below that it is sufficient to assume that $u$ is dominated from above by a stochastically ramified Dirichlet solution.

In particular, if $u$ is parabolic and bounded, and has a boundary limit at every boundary point accessible from above (or, more generally, we may exclude a boundary set of parabolic measure 0 , relative to $D$, identically on $D$ ), defining a boundary function $f$, then the theorem implies that $u$ is the Dirichlet solution on $D$ determined by the boundary function $f$. Later we shall strengthen this result by replacing the hypothesis that $u$ be bounded by the hypothesis that $|u|$ be dominated from above by a stochastically ramified Dirichlet solution.

Finally, we remark that, according to Theorem 3.3, the first assertion of Theorem 3.2 is true even if the boundary $C$ may contain irregular points. In fact, in Theorem 3.2, since $u$ is upper semicontinuous, $u$ is bounded from 
above on $D$, and the boundary function $f$ of that theorem is, at each point of the boundary of $D$, at least equal to the limit superior of $u$ on approach to the point from within $D$.

4. Martingales and parabolic functions. Let $D$ be a finite open set of $N+1$ space, and let $\{z(t), 0 \leqq t \leqq \infty\}$ be a Brownian trajectory process, with initial point in $D$. Let $\tau$ be the time a trajectory of the process first meets the boundary of $D$. The stochastic process $\left\{z^{*}(t), 0 \leqq t \leqq \infty\right\}$ defined by the equations

$$
z^{*}(t, \omega)= \begin{cases}z(t, \omega) & \text { if } t \leqq \tau(\omega), \\ z[\tau(\omega), \omega] & \text { if } t>\tau(\omega),\end{cases}
$$

will be called the $z(t)$ process stopped on the boundary of $D$. If $C$ is this boundary, we find that $z^{*}(\infty)=Z(z, C)$ with probability 1 .

THEOREM 4.1. Let $u$ be the Dirichlet solution for the heat equation on the finite open set $D$, with boundary function $f \in L(D)$, and define $u$ on the boundary as $f$. Then, if $\left\{z^{*}(t), 0 \leqq t \leqq \infty\right\}$ is a Brownian trajectory process with initial point in $D$, stopped on the boundary, the process $\left\{u\left[z^{*}(t)\right], 0 \leqq t \leqq \infty\right\}$ is a martingale.

By hypothesis, if $C$ is the boundary of $D, u$ is given by the equation

$$
u(z)=E\{u[Z(z, C)]\} .
$$

To prove that the $u\left[z^{*}(t)\right]$ process is a martingale, we need only prove that, if $0 \leqq s<t$,

$$
u\left[z^{*}(t)\right]=E\left\{u\left[z^{*}(\infty)\right] \mid z^{*}(s), s \leqq t\right\}
$$

with probability 1 . Since $z^{*}(t, \omega)=z^{*}(\infty, \omega)$, when $\tau(\omega) \leqq t$, this equation becomes a triviality in that case. On the other hand, if $\tau(\omega)>t, z^{*}(t, \omega)=z(t, \omega)$, and (4.2) then becomes, on this $\omega$ set,

$$
u[z(t)]=E\left\{u\left[Z\left(z_{0}, C\right)\right] \mid z(t)\right\} .
$$

Now the Brownian trajectories, after having gone for time $t$ without meeting $C$, proceed independently of their previous motion to $C$. The distribution of $Z\left(z_{0}, C\right)$ can therefore be computed as an average of that of $Z(z, C)$ over the distribution of $z=z(t)$ in $D$. That is, the right side of (4.3) becomes

$$
E\{u[Z(z(t), C)]\},
$$

which reduces the equation to be proved to the definition of $u$ as a Dirichlet solution, given in (4.1).

Let $u$ be defined and subparabolic on a finite open set $D$, and let $z$ be a point of this open set. Then we call "condition $H_{u}$ " the condition that, for every $z, u$ be a finite-valued right continuous function of the trajectory parameter of almost every Brownian trajectory of each Brownian trajectory proc- 
ess with initial point $z$ (excluding the parameter value 0 if $u(z)=-\infty$ ) as long as the trajectory remains in $D$. The exceptional set of trajectories may depend on $z$, and in fact the space on which the trajectory process is defined may vary with $z$. This condition is of course automatically satisfied if $u$ is continuous, in particular if $u$ is parabolic. It implies that $u$ is right continuous as a function of the trajectory parameter of almost every Brownian trajectory on the open parameter set for which the trajectory lies in $D$, for any Brownian trajectory process, regardless of the distribution of the initial point, which may not be confined to $D$ (always excluding the parameter value 0 if $u$ has the value $-\infty$ at the initial point of the trajectory).

It will be shown in $\$ 7$ that condition $H_{u}$ is always satisfied (Theorem 7.2). However it will be convenient to defer the proof of this fact, and to avoid misunderstanding we shall impose $H_{u}$ explicitly whenever it is needed, before its general validity is proved. But we stress that Theorem 7.2 means that this restriction is actually nugatory. If $u$ is subparabolic on a domain $D$, and if $\{z(t), 0 \leqq t \leqq \infty\}$ is a Brownian trajectory process with initial point in $D$, stopped on the boundary of $D$, then, under condition $H_{u}$, the stochastic process $\{u[z(t)], 0 \leqq t \leqq \infty\}$ is clearly separable, no matter how $u$ is defined on the boundary of $D$.

Lemma 4.2. Let $D$ be a finite open set, and let $D_{0}$ be an open set whose closure is a compact subset of $D$. Let $u$ be a function subparabolic on $D$. Then there is a function w, subparabolic on finite $N+1$ space, and a function $v$, of the form

$$
v(z)=\sum_{j=1}^{n} c_{j} \phi\left(z-z_{j}\right), \quad c_{j}>0,
$$

where $\phi$ is defined by (1.3), and where no $z_{j}$ lies in the closure of $D_{0}$, such that, if $u^{*}=v+w$,

(a) $u^{*}(z)=u(z)$ on $D_{0}$;

(b) $w$ is parabolic outside an arbitrarily small neighborhood of the closure of $D_{0}$;

(c) $\lim _{|z| \rightarrow \infty} w(z)=0$.

In proving the lemma, we shall suppose, enlarging $D_{0}$ slightly, if necessary, that $D_{0}$ is bounded by a finite number of polyhedra, with no horizontal faces. For simplicity, we shall treat only the case in which $D_{0}$ is the interior of a single such polyhedron $C_{0}$. It will be clear how to extend the argument to the general case. We can and shall also suppose that $u$ is continuous on $C_{0}$. (This step should have been but was not included in the proof of the corresponding result, Lemma 2.3, of [2].) In fact, we can suppose that the original set $D_{0}$ has been enlarged so that its boundary is at positive distance from the old one, if $u$ is not already continuous on the old one. We then, following Theorem 3.2, modify $u$ in a narrow ring region containing the new boundary, by replacing $u$ in the ring region by the Dirichlet solution for the 
ring, with $u$ as boundary function. This will leave $u$ subparabolic on $D$, if all the boundary points of the ring are regular, and will not change $u$ on the original $D_{0}$. However in this way we obtain a new $D_{0}$ and $C_{0}$, with $u$ continuous on $C_{0}$. Let $R$ be the finite complement of $D_{0} \cup C_{0}$. Let $C_{1}$ be a polyhedron, with interior $D_{1}$, lying in $D$, containing $C_{0}$ in its interior, and having no horizontal faces. Let $z_{1}, \cdots, z_{n}$ be points not on $C_{1}$, and with the property that every point of $C_{1}$ can be joined to some $z_{j}$ by a downward directed curve lying in $R$. Let $h$ be the solution of the Dirichlet problem for the heat equation, on the domain $R$, with boundary function 0 at $\infty$, and

$$
v_{1}(z)=\sum_{1}^{n} \phi\left(z-z_{j}\right)
$$

on $C_{0}$. Then $v_{1}(z)=h(z)=0$ for $z$ below both $C_{0}$ and the $z_{j}$ 's. If $\Delta=v_{1}-h,-\Delta$ is subparabolic on $R$, and has the boundary function 0 , as well as limit 0 when $|z| \rightarrow \infty$. Hence, according to Theorem 1.2, $\Delta \geqq 0$. Moreover, $\Delta$ is continuous on $C_{1}$, and if $\Delta$ vanished at $z \in C_{1}, \Delta$ would necessarily vanish at all points of $R$ which are endpoints of downward directed curves with initial point $z$, lying in $R$. But then $\Delta$ would vanish in a neighorhood of a $z_{j}$, which is impossible. Hence $\Delta$ is positive on $C_{1}$. Let $U$ be the solution of the Dirichlet problem for the heat equation on $R$, with boundary function 0 at $\infty$, and $u$ on $C_{0}$. Then $U$ is bounded, so that we can choose a constant $c$ so large that

$$
c \inf _{z_{1} \in C_{1}} \Delta(z)>u(z)-U(z) \quad \text { if } z \in C_{1} \text {. }
$$

This inequality must remain true for $z$ in some neighborhood of $C_{1}$. Define

$$
\begin{aligned}
& v(z)=c v_{1}(z), \\
& u^{*}(z)= \begin{cases}u(z) & \text { if } z \in D_{0} \cup C_{0}, \\
\operatorname{Max}[U(z)+c \Delta(z), u(z)] & \text { if } z \in D_{1} \cap R, \\
U(z)+c \Delta(z) & \text { if } z \notin D_{1},\end{cases}
\end{aligned}
$$

and set $w=u^{*}-v$. Then we shall show that $v, w$ satisfy the assertions of the lemma. Assertions (a), (b), (c) are easy to verify, so we confine ourselves to the proof that $w$ is subparabolic. Because of the choice of $c$,

$$
u^{*}(z)=U(z)+c \Delta(z)=U(z)-\operatorname{ch}(z)+v(z)
$$

on a neighborhood of $C_{1}$ as well as outside $D_{1}$. Thus $w$ is parabolic on a neighborhood of $C_{1}$ and outside $C_{1}$, that is, $w$ is parabolic on a domain slightly larger than the exterior of $D_{1}$. Between $C_{0}$ and $C_{1}, w$ is given by

$$
w(z)=\operatorname{Max}[U(z)-\operatorname{ch}(z), u(z)-v(z)]
$$

and is therefore subparabolic, as the maximum of two subparabolic functions. Since $w$ is obviously subparabolic on $D_{0}$, to finish the proof we need only con- 
sider the points of $C_{0}$. Let $z_{0}$ be a point of this set. Since $u$ is continuous at $z_{0}$, and since $z_{0}$ is a regular boundary point of $R, u^{*}$ and $w$ are also continuous at $z_{0}$. Since $u^{*}(z) \geqq u(z)$ on a small neighborhood of $z_{0}$, with equality at that point, $u^{*}$, as well as $u$, must have the average property of a subparabolic function in a neighborhood of $z_{0}$. Hence $u^{*}$ and therefore also $w$ are subparabolic on a neighborhood of $C_{0}$, as was to be proved.

THEOREM 4.3. Let $u$ be a function defined and subparabolic [parabolic] on a standard strip $a<s<b$, and suppose that, for some constant $c$, the condition $\left(1.6^{\prime}\right)[(1.6)]$ is satisfied. Let $z_{0}$ be any point in the strip, and let $\{z(t), 0 \leqq t \leqq \infty\}$ be a Brownian trajectory process, with initial point $z_{0}$, Then the stochastic process $\left\{u[z(t)], 0<t<s_{0}-a\right\}$, where $s_{0}=\operatorname{ord}\left(z_{0}\right)$, is a semimartingale [martingale]. If $u\left(z_{0}\right)>-\infty$, the point 0 can be added to the parameter set in the conclusion.

In the following, the integral will converge absolutely, in view of $\left(1.6^{\prime}\right)$ or (1.6). Let $\mathcal{F}(s)$ be the smallest Borel field of $\omega$ sets containing all sets of probability 0 , and such that every $z(t)$ with $t \leqq s$ is measurable relative to $\mathcal{F}(s)$. We shall prove that the $u[z(t)]$ process is a semimartingale or martingale relative to the $\mathcal{f}(s)$ family of fields. We have, in the semimartingale case, in view of (1.5) with inequality, if $0 \leqq s<t$, and if we write $\left(\xi, s_{0}-s\right)$ for $z(s)$,

$$
\begin{aligned}
& E\{u[z(t)] \mid \mathcal{F}(s)\} \\
& \quad=E\{u[z(t)] \mid z(s)\} \\
& \quad=\frac{\int_{-\infty}^{\infty} \cdots \int_{-\infty}^{\infty} \exp \left(-\sum_{1}^{N}\left(\eta_{j}-\xi_{j}\right)^{2} / 4(t-s)\right) u\left(\eta, s_{0}-t\right)}{2^{N}[\pi(t-s)]^{N / 2}} d \eta_{1} \cdots d \eta_{N} \\
& \geqq u\left(\xi, s_{0}-s\right),
\end{aligned}
$$

as was to be proved. In the martingale case, there is equality here. If $u\left(z_{0}\right)$ $=-\infty$, we exclude the parameter value $s=0$, since this makes $u[z(0)]=-\infty$ and we are not considering semimartingales with infinite expectations in this paper.

THEOREM 4.4. Let $u$ be a function subparabolic [parabolic] on a finite open set $D$, and satisfying condition $H_{u}$ there. Let $D_{0}$ be an open set whose closure is compact and lies in $D$, let $z_{0} \in D_{0}$, and let $\left\{z^{*}(t), 0 \leqq t \leqq \infty\right\}$ be a Brownian trajectory process with initial point $z_{0}$, stopped on the boundary of $D_{0}$. Then the stochastic process $\left\{u\left[z^{*}(t)\right], 0<t \leqq \infty\right\}$ is a semimartingale [martingale]. If $u\left(z_{0}\right)>-\infty$, the point 0 can be added to the parameter interval in this assertion.

It will be proved below, using this result, that the hypotheses can be weakened considerably. We can take $D_{0}=D$, if we suppose that $u[|u|]$ is bounded from above by stochastically ramified Dirichlet solution, and if $u\left[z^{*}(\infty)\right]$ is defined as a boundary limit along Brownian trajectories. We shall show that such a boundary limit function exists. 
To prove the theorem as stated, suppose first that $u$ is subparabolic, and that $u\left(z_{0}\right)$ is finite. Let $u^{*}, v, w$ be the functions defined in Lemma 4.2. Since there are only finitely many $z_{j}$ 's, almost no Brownian trajectory from $z_{0}$ passes through a $z_{j}$. Hence almost all sample functions of the $v[z(t)]$ process are finite valued and right continuous, and, indeed are continuous except at finitely many parameter points. According to our definition, $w$ is continuous on the complement of a slightly larger set than $D_{0}$. Hence $u^{*}$ and $w$ can be supposed right continuous on almost all Brownian trajectories from $z_{0}$. Since $v$ is parabolic on $D_{0}$, and continuous on the closure of $D_{0}$, it is a Dirichlet solution on $D_{0}$, determined by $v$ on $C_{0}$ as boundary function, according to Theorem 3.3, applied to $v$ and $-v$. Hence, according to Theorem 4.1, the process $\left\{v\left[z^{*}(t)\right], 0 \leqq t \leqq \infty\right\}$ is a martingale. According to Theorem 4.3, if $\{z(t)$, $0 \leqq t \leqq \infty\}$ is the process which, when stopped, yields the $z^{*}(t)$ process, the process $\{w[z(t)], 0 \leqq t<\infty\}$ is a semimartingale. Then the stopped process $\left\{w\left[z^{*}(t)\right], 0 \leqq t \leqq \infty\right\}$ is also a semimartingale. As these processes are semimartingales relative to the same families of fields, it follows that the sum process

$$
\left\{u^{*}\left[z^{*}(t)\right], 0 \leqq t \leqq \infty\right\}=\left\{u\left[z^{*}(t)\right], 0 \leqq t \leqq \infty\right\}
$$

is a semimartingale. If $u\left(z_{0}\right)=-\infty, u$ can be replaced in a small open sphere containing $z_{0}$ by the Dirichlet solution for the heat equation in the sphere, with boundary function $u$ on the surface, thus reducing the problem to that already considered, but in that case the parameter value 0 will have to be omitted from the conclusion. If $u$ is parabolic, what we have proved can be applied to $u$ and $-u$ to derive the stated result in the parabolic case.

Let $u$ be a function subparabolic on an open set $D$, and suppose that $u$ satisfies condition $H_{u}$ there. Let $z_{0}$ be a point of $D$, and, for each number $t$ in some linear set $T$, let $D_{t}$ be an open set containing $z_{0}$, whose closure is a compact subset of $D$, with $D_{s} \subset D_{t}$ when $s<t$. Let $\{z(t), 0 \leqq t \leqq \infty\}$ be a Brownian trajectory process with initial point $z_{0}$, and let $\tau_{t}$ be the time a Brownian trajectory from $z_{0}$ first meets $D_{t}$. Then $\left\{u\left[z\left(\tau_{t}\right)\right], t \in T\right\}$ is a stochastic process which we now prove is a semimartingale. The proof will also demonstrate that, if $u$ is parabolic, the process is a martingale. If $u\left(z_{0}\right)$ is finite, we can assume that $T$ has a first point $t_{0}$, with $\tau_{t_{0}}=0$, so that $u\left[z\left(\tau_{t_{0}}\right)\right]$ $=u\left(z_{0}\right)$, and the assertion remains true. The assertion is an almost immediate consequence of Theorem 4.4, because, for fixed $s$, if $\left\{z^{*}(t), 0 \leqq t \leqq \infty\right\}$ is the $z(t)$ process stopped at time $\tau_{s}$, the $u\left[z^{*}(t)\right]$ process is a semimartingale, according to Theorem 4.4, and the process $\left\{u\left[z\left(\tau_{t}\right)\right], t<s\right\}$ is obtained from this process by optional sampling, and hence is a semimartingale, according to the general semimartingale theorems of [1] as long as $E\left\{u\left[z^{*}(t)\right]\right\}$ is bounded from below. A possible lower bound is $u\left(z_{0}\right)=E\left\{u\left[z^{*}(0)\right]\right\}$, if $u\left(z_{0}\right)$ is finite. Otherwise, the same device used in the proof of Theorem 4.4 to take care of the case $u\left(z_{0}\right)=-\infty$ can be applied here. One implication of our assertion that we have now proved is that $E\left\{u\left[z\left(\tau_{t}\right)\right]\right\}$ does not decrease when $t$ increases. In particular, if $T$ has only two points, we find that the parabolic 
average of a subparabolic function relative to an open set, whose closure is a compact subset of its domain of definition, at a specified point, does not decrease when the set increases. We shall see that this result can be extended to unbounded sets, and thus the corresponding well-known result for subharmonic functions, involving harmonic instead of parabolic measure, can be considered a special case. Finally, we remark that if the functions considered in this paragraph are parabolic, the corresponding stochastic processes are martingales.

5. The boundary limits of subparabolic and parabolic functions. Throughout this section, we shall deal with a subparabolic or parabolic function $u$, defined on a finite open set $D$, with boundary $C$, under various restrictions. In the subparabolic case, we suppose that condition $H_{u}$ is satisfied. The following technique will be useful. Let $D_{1} \subset D_{2} \subset \ldots$ be a sequence of open sets whose union is $D$, and whose closures are compact subsets of $D$. Let $\left\{z_{z}(t)\right.$, $0 \leqq t \leqq \infty\}$ be a Brownian trajectory process with initial point $z$ in $D$. (We shall omit the subscript $z$ here and in the following if the omission will cause no confusion.) Let $\tau_{n}\left[\tau_{\infty}\right]$ be the time a trajectory of this process first meets the boundary of $D_{n}[D]$. Let $\left\{z_{z}{ }^{*}(t), 0 \leqq t \leqq \infty\right\}$ be the $z_{z}(t)$ process stopped on the boundary of $D$. According to our discussion at the end of $\S 4$, the stochastic process $\left\{u\left[z_{z}\left(\tau_{n}\right)\right], 1 \leqq n<\infty\right\}$ is a semimartingale, or a martingale if $u$ is parabolic, where it is to be understood throughout that, in spite of the notation, the values of $n$ for which $D_{n}$ does not contain the point $z$ are to be ignored. The properties of this stochastic process will be used in our analysis of the boundary limits of $u$. We note that, if $u$ is the Dirichlet solution on $D$ determined by the boundary function $f$, and if $u$ is defined on $C$ as $f$, then the process $\left\{u\left[z_{z}\left(\tau_{n}\right)\right], 1 \leqq n \leqq \infty\right\}$ is a martingale. This fact can be deduced from Theorem 4.1 , by applying optional sampling to the martingale $\left\{u\left[z_{z}{ }^{*}(t)\right]\right.$, $0 \leqq t \leqq \infty\}$, sampling it at times $\tau_{1}, \tau_{2}, \cdots, \tau_{\infty}$, or the method of proof of Theorem 4.1 can also be used to deduce this closely related result. A very slightly stronger assertion will be useful below. The second method of proof just suggested yields the fact that, if $\mathcal{F}_{n}$ is the smallest Borel field of $\omega$ sets, containing all sets of measure 0 , with respect to which $z_{z}\left(\tau_{m}\right)$ is measurable, for all $m \leqq n$, then $\mathcal{F}_{\infty}$ is the smallest Borel field containing $\cup_{n<\infty} \mathcal{F}_{n}$, and the $u\left[z_{z}\left(\tau_{n}\right)\right]$ process is a martingale relative to this sequence of fields, that is,

$$
u\left[z_{z}\left(\tau_{n}\right)\right]=E\left\{f\left[z_{s}\left(\tau_{\infty}\right)\right] \mid \mathcal{F}_{n}\right\},
$$

with probability 1 .

In the following, we shall say that $u$ is dominated from above by a positive parabolic function $v$ if there is a positive parabolic function $v$, with the same domain of definition as $u$, and satisfying the inequality $u \leqq v$.

THEOREM 5.1. Let $u$ be a function subparabolic on a finite open set D. Suppose that $u$ satisfies condition $H_{u}$ there, and that $u$ is dominated from above by $a$ 
positive parabolic function. Then $u$ has a finite limit along almost every Brownian trajectory from any point of $D$ to the boundary.

Note that, if the point $\infty$ has positive parabolic measure relative to $D$ at a point of $D$, then this theorem makes the nontrivial assertion that $u$ has a finite limit along almost every Brownian trajectory which goes from that point to $\infty$ without meeting the boundary of $D$ in a finite point.

The proof of this theorem is precisely the same as proofs given in corresponding situations in two previous papers (see [2, Theorem 6.1 ], and, in more elegant form [3, Theorem 8.3], where the proof is based on the key Theorem 1.2), and will be omitted. It is gratifying that, according to the following theorem, the boundary limit which we have now shown to exist is the prescribed boundary value, if $u$ is a Dirichlet solution.

THEOREM 5.2. If $u$ is the Dirichlet solution for the heat equation on the finite open set $D$, with boundary function $f \in L(D)$, then $u$ has the limit $f$ along almost every Brownian trajectory from any point of $D$ to the boundary.

Theorem 5.1 is applicable, because, if $C$ is the boundary of $D$,

$$
u(z)=E\{f[Z(z, C)]\} \leqq E\{|f[Z(z, C)]|\},
$$

and the function defined by the expectation on the right is non-negative and parabolic on $D$. Thus $u$ actually has a boundary limit along almost every Brownian trajectory from a point of $D$ to the boundary. To identify the boundary limit function with $f$, we apply the technique and notation of the first paragraph of this section, to prove that

$$
\lim _{n \rightarrow \infty} u\left[z_{z}\left(\tau_{n}\right)\right]=f\left[z_{z}\left(\tau_{\infty}\right)\right]
$$

with probability 1 . According to general martingale theory, it follows from (5.1) that

$$
\lim _{n \rightarrow \infty} u\left[z_{z}\left(\tau_{n}\right)\right]=E\left\{f\left[z_{z}\left(\tau_{\infty}\right) \mid \mathcal{F}_{\infty}\right\}\right.
$$

with probability 1 . The right side is, with probability 1 , equal to the asserted limit $f\left[z_{z}\left(\tau_{\infty}\right)\right.$ ] because the latter random variable is measurable with respect to $\mathcal{F}_{\infty}$.

As an example of this theorem, we consider the function $u_{A, D}$ discussed in Theorem 2.2, under the hypothesis that $A$ is closed in the unextended topology. We have seen that, on $D-A, u_{A, D}$ is the solution of the Dirichlet problem for the heat equation, with boundary function value 1 on the part of the boundary on $A$, and 0 on the boundary of $D$. Hence, according to Theorem 5.2, $u_{A, D}$ has the limit 1 on almost every Brownian trajectory from a point of $D-A$ to $A$, the limit 0 on almost every Brownian trajectory from a point of $D-A$ to the boundary of $D$. Applying Theorem 3.1, we find that, at the regular boundary points of $D-A$ on $A, u_{A, D}$ has the value 1 , by definition 
of this function. The function is continuous at each such point because $u_{A, D}$ is lower semicontinuous and less than or equal to 1 . At an irregular boundary point of $D-A$ on $A, u_{A, D}$ may take on a value less than 1 , even though the boundary limit along a Brownian trajectory is 1 . For example, if $N=1$ and if $A$ is a compact horizontal line segment, $u_{A}$ vanishes at every point of $A$, even though any boundary limit along a Brownian trajectory to an interior point of the segment is 1 .

We observe that the situation in Theorem 5.1, parabolic case, is more general than that in Theorem 5.2. In the first place, it is easy to give examples in which $u$ is parabolic on $D$, and positive, but with limit 0 at the boundary along almost every Brownian trajectory from a point of $D$, so that $u$ is not a Dirichlet solution. For example, we shall see that, if $N=1,(5.8)$, with $\psi$ a singular bounded monotone nondecreasing function, defines such a parabolic function $u$, with domain the upper half-plane. In the second place, even if the initial point of the Brownian trajectories in Theorem 5.1 is held fast, the limit values at the boundary define a random variable, that is, an $\omega$ function, which may not in turn define a single-valued function on the boundary.

In order to proceed further in this analysis, it will be convenient to generalize the notion of a Dirichlet solution for the heat equation. Whatever generalization one gives involves some manner in which a parabolic function is to have prescribed boundary limits, thereby defining a boundary function in some generalized sense. The Dirichlet problem then consists of the discovery of a parabolic function with the prescribed boundary function. In this rather vaguely described procedure, one obtains a class of generalized Dirichlet solutions. If the theory is to be an elegant one, it must be possible to give a reasonably simple characterization of a Dirichlet solution, in terms of its regularity properties in its domain, which is not just a rewording of the definition, and which implies the existence of the generalized boundary function which is to determine it as a Dirichlet solution.

In view of Theorems 5.1 and 5.2, it appears that a natural way to approach the boundary is along Brownian trajectories. This type of approach leads to a new definition of a boundary function, which we now proceed to give. This definition seems artificial at first, artificially designed to conform to probability notions, but it appears to be the one most adapted to ordinary function theoretic notions also. We shall see that it leads to a theory satisfying the criterion of elegance given in the preceding paragraph.

We use the notation of the first paragraph of this section. Let $\Omega_{z}$ be the measure space on which the $z_{z}(t)$ process is defined. This space may depend on $z$. Suppose that, to each point $z$ in $D$, there corresponds a random variable $f(z)$, defined on $\Omega_{z}$, and that the family of random variables $\{f(z), z \in D\}$ has the following properties.

(a) Let $D_{0}$ be any open set, whose closure is a compact subset of $D$, let $z$ be a point of $D_{0}$, and let $\tau$ be the time a $z_{z}^{*}(t)$ process trajectory first meets the 
boundary of $D_{0}$. Then $f(z)$ is a function of $z_{z}^{*}(t)$ for $t \geqq \tau$. More precisely, in the language of $[1], f(z)$ is measurable on the sample space of the random variables $\left\{z_{z}^{*}(\tau+t), t \geqq 0\right\}$.

(b) If $D_{0}$ is as in (a), the distribution of $f(z)$ is the parabolic average relative to $D_{0}$ of that of $f(w)$, for $w$ on the boundary of $D_{0}$. That is, if $B$ is a linear Borel set, the probability that the value of $f(z)$ lies in $B$ defines a parabolic function of $z$.

Then the family $\{f(z), z \in D\}$, which we shall sometimes denote simply by $f$ below, will be called a stochastic boundary function. It is clear that, if $u$ is any Baire function defined on $D$, and if for each $z$ in $D, u$ has a limit along almost every Brownian trajectory from $z$ to the boundary of $D$, then the limit $f(z)$ is a random variable which defines a stochastic boundary function. Theorems 5.1 and 5.2 exhibit the importance of the concept of a stochastic boundary function for the theory of subparabolic and parabolic functions, and thereby for the more special theory of subharmonic and harmonic functions. In the latter case, a special and independent definition can be made, in an obvious way, without involving the time variable. The point is that each type of approach to a boundary on probability trajectories leads to a corresponding definition of a stochastic boundary function.

If $g$ is an ordinary single-valued Baire function on the boundary of $D$, it determines a corresponding stochastic boundary function $f$, by way of the definition

$$
f(z)=g\left[z_{z}^{*}(\infty)\right]
$$

In this sense, an ordinary boundary function can be considered as a special case of a stochastic boundary function. To see how this procedure can be generalized to ramified boundary functions, we first examine a very simple case. Suppose that $N=1$, and that $D$ is the finite complement of a compact line segment. Then the boundary $C$ consists of the line segment and the point $\infty$. We distinguish the two sides of the line segment, except at the endpoints (prime end theory) and suppose that $g$ is a single valued Baire function on this ramified boundary. Then the above equation (5.2) again defines a corresponding stochastic boundary function, if $z_{z}^{*}(\infty)$ is determined as on the one or other side of the line segment depending on the side of approach of the Brownian trajectory. It is clear that this procedure will be available for many systems of ramification, so that the generalized Dirichlet solutions we have defined solve the Dirichlet problem for ramified boundaries, as long as the ramification of the boundary leads to the following situation. The set $D$ is homeomorphic to a dense open subset $D^{\prime}$ of a new topological space, the remainder of the new space being $C^{\prime}$, the ramified boundary. If $L$ is a downward directed curve, in $D$ except for the endpoint, which is on $C$, the curve less the endpoint corresponds, in the new topological space, to a continuous curve in $D^{\prime}$, approaching as a limit a unique point $L^{\prime}$ of $C^{\prime}$. Thus there is a 
many to one map of the boundary points of $D$ which are accessible from above into points of $C^{\prime}$. If to the point $\omega$ determining a Brownian trajectory $L$ ending at $z_{*}^{*}(\infty, \omega)$ we make correspond the unique image $L^{\prime}$, a function on the ramified boundary $C^{\prime}$ will determine a function $f(z)$ on $\Omega_{z}$, if in (5.2) we interpret $g\left[z_{z}^{*}(\infty)\right]$ as $g\left(L^{\prime}\right)$. It would be interesting to make a full and rigorous analysis of this situation, unnecessary for the purposes of this paper, to obtain a boundary ramification in purely topological terms which matches the stochastic ramification so that stochastic boundary functions become single valued functions on the ramified boundary.

If $f$ is a stochastic boundary function, we shall say that it is finite with probability 1 if, for each $z$ in $D, f(z)$ is finite with probability 1 . We write $f \in L(z, D)$ if $E\{|f(z)|\}$ is finite, where the expectation is that relating to $\Omega_{z}$, and we write $f \in L(D)$ if $f \in L(z, D)$ for every $z$ in $D$. Then it is clear that, if $f \in L(D)$, the function $u$ defined on $D$ by the equation

$$
u(z)=E\{f(z)\}
$$

is parabolic. We shall call this function the stochastically ramified Dirichlet solution corresponding to the stochastic boundary function $f$.

Our generalizations of the concepts of boundary function and Dirichlet solution lead in part to mere rephrasing of old theorems, but also to interesting new ones. Theorem 4.1 remains true for stochastically ramified Dirichlet solutions, if we do not define $u$ on the boundary, but replace $u\left[z^{*}(\infty)\right]$ in the statement of the theorem by $f(z)$, the element of the stochastic boundary function. The proof needs no change. Theorem 5.1 states that a subparabolic function dominated from above by a positive parabolic function has a stochastic boundary function which is finite with probability 1 . Theorem 5.2 remains true, with no change in proof, if $f$ is a stochastic boundary function and if $u$ is the corresponding stochastically ramified Dirichlet solution. The reinterpretation of our old results is thus trivial.

The following theorem is a far-reaching generalization of parts of Theorems $1.2,3.2,3.3,4.4$, in all of which the function $u$ in question is bounded from above. This condition on $u$ is now replaced by the weaker condition that $u$ be dominated from above by a positive stochastically ramified Dirichlet solution. The latter condition will be put into another form below. In addition, other conditions in some of the earlier theorems will be dropped entirely. We observe that every stochastically ramified Dirichlet solution is dominated from above by a positive such solution, for example that having as stochastic boundary function the absolute value of the stochastic boundary function of the given solution. We use the notation of the first paragraph of this section in the statement of the following theorem.

TheоReм 5.3. Let $u$ be a function subparabolic on a finite open set D. Suppose that $u$ satisfies condition $H_{u}$ and is dominated from above by a positive stochastically ramified Dirichlet solution. Let $f$ be the stochastic boundary func- 
tion of $u$. Then $f \in L(D)$, and, if $u_{1}$ is the stochastically ramified Dirichlet solution determined by $f, u \leqq u_{1}$. Moreover, if $z \in D$, the stochastic processes

$$
\left\{u\left[z_{z}^{*}(t)\right], 0<t \leqq \infty\right\}, \quad\left\{u\left[\left(z_{s}\left(\tau_{n}\right)\right], 1 \leqq n \leqq \infty\right\}\right.
$$

are semimartingales, if we replace $u$ on the boundary of $D$, where such a term appears in (5.3), by $f(z)$. If $u(z)>-\infty$, the parameter value $t=0$ can be adjoined to the parameter set in the first process of (5.3).

Since $u$ is not defined on the boundary $C$ of $D, u\left[z\left(\tau_{\infty}\right)\right]$ is not intrinsically defined. The penultimate sentence of the theorem means that this undefined symbol is to be replaced in (5.3) by $f(z)$. Similarly, $u\left[z_{z}^{*}(t, \omega)\right]$ is not defined if $z_{z}^{*}(t, \omega)$ is a point of $C$, and again the value to be used is the corresponding value of $f(z)$.

We find, repeating the type of reasoning we have gone through before, that it will suffice, in proving that the first process in (5.3) is a semimartingale, to prove

$$
u(z) \leqq E\left\{u\left[z_{z}^{*}(s)\right]\right\}, \quad 0<s \leqq \infty,
$$

under the hypothesis that the left side of the inequality is inite. This inequality, for $s=\infty$, if interpreted in the obvious way, and taken in conjunction with the fact that $u\left[z_{z}^{*}(\infty)\right] \leqq v\left[z_{z}^{*}(\infty)\right]$, where $v$ is a stochastically ramified Dirichlet solution, also implies that $f \in L(z, D)$, and that $u(z) \leqq u_{1}(z)$. Fixing $z$, let $\left\{{ }_{n} z(t), 0 \leqq t \leqq \infty\right\}$ be the $z_{z}^{*}(t)$ process stopped on the boundary. of $D_{n}$. Then the $u\left[{ }_{n} z(t)\right]$ process is a semimartingale, according to Theorem 4.4 , so that

$$
u(z) \leqq E\left\{u\left[{ }_{n} z(s)\right]\right\} .
$$

Moreover, according to our definitions,

$$
\lim _{n \rightarrow \infty} u\left[{ }_{n} z(s)\right]=u\left[z^{*}(s)\right],
$$

with probability 1 . Now if $u \leqq 0$, and if $n \rightarrow \infty$ in (5.5), we find, applying Fatou's lemma, that (5.4) is true. If this restriction is not imposed on $u$, but if we impose only the restriction that $u$ be dominated from above by a positive stochastically ramified Dirichlet solution $v$, we find, applying the result already obtained to $u-v$, that

$$
u(z)-v(z) \leqq E\left\{u\left[z^{*}(s)\right]-v\left[z^{*}(s)\right]\right\} .
$$

Since, according to our generalization of Theorem 5.2, the $v\left[z^{*}(t)\right]$ process is a martingale,

$$
E\left\{v\left[z^{*}(s)\right]\right\}=E\left\{v\left[z^{*}(0)\right]\right\}=v(z),
$$

so that the preceding inequality reduces to (5.4). Finally, the $u\left[z_{z}\left(\tau_{n}\right)\right]$ process can be treated in the same way, or the assertion of the theorem about it can 
be deduced from the first assertion, which we have now proved, by applying optional sampling, just as in the particular case when $u$ is an ordinary Dirichlet solution, which was considered in the first paragraph of this section.

CoROLlary 1. Let $u$ be a stochastically ramified Dirichlet solution on a finite open set $D$. Then $u$ is such a solution on every open subset of $D$.

In fact, applying Theorem 5.3 to both $u$ and $-u$, we find that the first process in (5.3) is a martingale. Let $D^{\prime}$ be an open subset of $D$, and let $z$ be a point of $D^{\prime}$. Then, if the martingale is stopped on the boundary of $D^{\prime}$, we obtain a new martingale $\left\{u\left[z_{z}^{\prime}(t)\right], 0 \leqq t \leqq \infty\right\}$, where we make the same convention as before on the interpretation of $u$ on the boundary. Writing down the equality of the expectations of the first and last random variables of this martingale, we find that

$$
u(z)=u\left[z_{z}^{\prime}(0)\right]=E\left\{u\left[z_{z}^{\prime}(\infty)\right]\right\},
$$

and this is precisely the definition of $u$ as a stochastically ramified Dirichlet solution on $D^{\prime}$.

In Theorem 5.3, let $v$ be a positive stochastically ramified Dirichlet solution dominating $u$. Let $D^{\prime}$ be any open subset of $D$, and let $f^{\prime}\left[g^{\prime}\right]$ be the stochastic boundary function of $u[v]$ on the boundary of $D^{\prime}$. Then $f^{\prime}$ and $g^{\prime}$ are in the class $L(D)$, according to Theorem 5.3 and its first corollary. Let $u^{\prime}$ be the stochastically ramified Dirichlet solution on $D^{\prime}$ determined by the stochastic boundary function $f^{\prime}$. Then $u \leqq u^{\prime}$ on $D^{\prime}$, according to Theorem 5.3. Moreover, if, in defining $f^{\prime}$, we replace the limit value of $u$ along a Brownian trajectory to a point of the boundary of $D^{\prime}$ which lies in $D$ by the value of $u$ at that point, we would have a new stochastic boundary function $f^{\prime \prime}$, and, in view of the upper semicontinuity of $u, f^{\prime} \leqq f^{\prime \prime} \leqq g^{\prime}$. Hence $f^{\prime \prime} \in L(D)$, and the Dirichlet solution on $D^{\prime}$ determined by $f^{\prime \prime}$ is at least equal to $u$ on $D^{\prime}$. We summarize these results in the following somewhat loosely phrased statement, which we write as a second corollary to Theorem 5.3. This corollary makes it clear how we have now generalized such theorems as Theorem 3.2.

COROLlARY 2. Let $u$ be a function subparabolic, satisfying condition $H_{u}$, and dominated from above by a positive stochastically ramified Dirichlet solution, on a finite open set $D$. Then the parabolic average of $u$ at $z$, relative to an open subset $D^{\prime}$ of $D$ which contains $z$, does not decrease when $D^{\prime}$ increases.

It is to be understood that, in computing parabolic averages, we may need the stochastic boundary function of $u$ on the boundary of $D$, if $D$ and $D^{\prime}$ have common boundary points, and that either the limits along Brownian trajectories or the values of $u$ can be used at boundary points of $D^{\prime}$ in $D$. In particular, this corollary implies a result we actually proved in the course of proving Corollary 1 , but did not state explicitly, that a stochastically ramified Dirichlet solution is unchanged if replaced on any open subset of its domain of definition by the stochastically ramified Dirichlet solution deter- 
mined by itself or its own stochastic boundary function on the boundary.

The following theorem characterizes the functions to which Theorem 5.1 is applicable.

TheOREM 5.4. Let $u$ be a function subparabolic on a finite open set $D$, and suppose that $u$ satisfies condition $H_{u}$. Then, if $u$ is dominated from above by $a$ positive parabolic function, the inequality

$$
\sup _{n} E\left\{\left|u\left[z_{z}\left(\tau_{n}\right)\right]\right|\right\}<\infty
$$

is satisfied for all $z$ in $D$. Conversely, if (5.6) is true for $z$ on a subset of $D$ which scans $D, u$ is so dominated.

In particular, if $u$ is parabolic, it is dominated by a positive parabolic function if and only if $|u|$ is so dominated. In fact (5.6) is unchanged if $u$ is replaced by $-u$, or, more directly, if $u$ is so dominated by $v$,

$$
|u| \leqq 2 v-u,
$$

and the right side of this inequality is a positive parabolic function.

To prove the theorem, suppose first that $u$ is dominated by the positive parabolic function $v$. Then

$$
E\left\{\left|u\left[z_{z}\left(\tau_{n}\right)\right]\right|\right\} \leqq 2 E\left\{v\left[z_{z}\left(\tau_{n}\right)\right]\right\}-E\left\{u\left[z_{z}\left(\tau_{n}\right)\right]\right\} .
$$

Since the $v\left[z_{z}\left(\tau_{n}\right)\right]$ process is a martingale, the first expectation on the right does not depend on $n$. Since the $u\left[z_{z}\left(\tau_{n}\right)\right]$ process is a semimartingale, the second term on the right (including the negative sign) does not increase when $n$ increases. Hence (5.6) is true. Conversely, suppose that (5.6) is true on a $z$ set which scans $D$. In proving the truth of the converse, we can assume that $u \geqq 1$, replacing $u$ by $\max [u, 1]$ if this inequality is not true already. Define $v_{n}$ as the Dirichlet solution on $D_{n}$ with $u$ as boundary function. Then, according to Theorem 5.3, $u \leqq v_{n}$ un $D_{n}$, and, according to Corollary 2 of the same theorem, the sequence $\left\{v_{n}, n \geqq 1\right\}$ is monotone nondecreasing. Hence there is a positive limit $v$, with $u \leqq v$ on $D$. The function $v$ is finite on a set which scans $D$, since (5.6) is true on such a set, and $v$ is therefore parabolic, and is the desired positive parabolic function which dominates $u$ from above.

It is gratifying that there is a simple necessary and sufficient condition on a parabolic function that it be a Dirichlet solution, which does not involve a boundary function. The following theorem provides such a condition, and we shall show that it reduces to a well-known classical condition for harmonic functions defined on spheres.

THEOREM 5.5. Let $u$ be a function parabolic on a finite open set $D$. Then, if $u$ is a stochastically ramified Dirichlet solution, the sequence $\left\{u\left[z_{z}\left(\tau_{n}\right)\right], 1 \leqq n<\infty\right\}$ is uniformly integrable, for each point $z$ in $D$. Conversely, if the uniform integrability condition is satisfied for each point $z$ in a subset of $D$ which scans $D$, then $u$ is a stochastically ramified Dirichlet solution. 
In other words, both in Theorems 5.4 and 5.5 we consider $u$ on the boundary of $D_{n}$, relative to the parabolic measure at $z$, of boundary sets. The condition of Theorem 5.4 is that the integral of $|u|$ with respect to the measure be bounded independently of $n$; that of Theorem 5.5 is the stronger condition of uniform integrability. In both cases, the conditions are stated for a fixed sequence of nested open sets $\left\{D_{n}\right\}$ and for each point of a $z$ set which scans $D$. In particular, according to Theorem 5.5, every bounded parabolic function is a stochastically ramified Dirichlet solution.

To prove the theorem we suppose first that $u$ is a stochastically ramified Dirichlet solution. Applying Theorem 5.3, we find that the process $\left\{u\left[z_{z}\left(\tau_{n}\right)\right]\right.$, $1 \leqq n \leqq \infty\}$ is a martingale, if the last random variable is interpreted properly. But then, according to a standard martingale theorem, these random variables are uniformly integrable. Conversely, suppose that $u$ is parabolic, let $A$ be the set of values of $z$ in $D$ at which the sequence $\left\{u\left[z_{z}\left(\tau_{n}\right)\right], 1 \leqq n<\infty\right\}$ is uniformly integrable, and suppose that $A$ scans $D$. Fix $z$ in $A$ and denote the $n$th random variable of the sequence by $y_{n}$. Then this sequence is a martingale whose random variables are uniformly integrable and hence $\sup _{n} E\left\{\left|y_{n}\right|\right\}<\infty$. According to a standard martingale theorem, the sequence is convergent with probability 1 to some random variable $y_{\infty}$. Applying Theorem 5.4, we find that the hypotheses of Theorem 5.1 are satisfied, so that $u$ has a stochastic boundary function $f$, in the class $L(D)$, and, obviously, $f(z)=y_{\infty}$, with probability 1 . Moreover, according to standard martingale theory, the process $\left\{y_{n}, 1 \leqq n \leqq \infty\right\}$ is a martingale. There would be no change whatever in any of the reasoning if a constant random variable, $y_{0}=u(z)$, were adjoined to the $y_{n}$ process, preceding the other random variables of the process. But then, equating expectations of $y_{0}$ and $y_{\infty}$, we have

$$
E\left\{y_{0}\right\}=u(z)=E\left\{y_{\infty}\right\}=E\{f(z)\} .
$$

That is, $u$ on $A$ is the stochastically ramified Dirichlet solution $u_{1}$ determined by the stochastic boundary function $f$. To finish the proof, we prove that $A=D$. From martingale theory we know that, even if $z$ is not in $A$,

$$
E\left\{\left|y_{1}\right|\right\} \leqq E\left\{\left|y_{2}\right|\right\} \leqq \cdots \text {. }
$$

Moreover, by Fatou's lemma,

$$
E\left\{\left|y_{\infty}\right|\right\} \leqq \lim _{n \rightarrow \infty} E\left\{\left|y_{n}\right|\right\}
$$

and there is equality if and only if the $y_{n}$ sequence is uniformly integrable. Now $E\left\{\left|y_{n}\right|\right\}$ defines a parabolic function of $z$ on $D_{n}$, in fact a certain Dirichlet solution, and $E\left\{\left|y_{\infty}\right|\right\}$ defines a parabolic function on $D$. Thus the two sides of (5.7) define parabolic functions on $D$, and $A$ is the set on which their difference, a non-negative parabolic function, vanishes. Since $A$ scans $D$, $A=D$, as was to be proved.

The following corollary is an obvious consequence of the theorem. 
COROLlaRy. If $u$ is a parabolic function, and if $|u|$ is dominated from above by $a$ positive stochastically ramified Dirichlet solution, then $u$ is itself $a$ stochastically ramified Dirichlet solution.

It will sometimes be convenient to use unbounded open sets $D_{n}$ in the criteria of Theorems 5.4 and 5.5, although this is impossible as they are now stated. In view of the results we have obtained, we can extend these theorems, imposing no conditions on these open sets except that $D_{1} \subset D_{2} \subset \cdots$ and $\cup_{1}^{\infty} D_{n}=D$, if we add-appropriate conditions relating $u$ and each $D_{n}$. Theorem 5.4 will remain true using these more general nested sets, and the proof will need no change whatever if, for each $n$, we suppose that $u$ on $D_{n}$ is dominated from above by a positive stochastically ramified Dirichlet solution. Theorem 5.5 will remain true, and the proof will need no change, if we suppose that $u$ on $D_{n}$ is a stochastically ramified Dirichlet solution. These hypotheses are necessarily satisfied if, as we have supposed heretofore, the closure of each $D_{n}$ is a compact subset of $D$. However these hypotheses represent real restrictions on $u$ if the $D_{n}$ 's are large, and the theorems now become trivial if the $D_{n}$ 's are too large, for example if $D_{1}=D$.

The sufficient conditions we have found in Theorems 5.4 and 5.5 are unwieldy, because they must be checked at each point of a set which scans $D$. However, in simple cases the checking problem may have an elegant formulation. As a first example of the application of these theorems, we consider an extreme case, in which the results are well known. Let $D$ be the direct product of a solid $N$ sphere of unit radius, center at the origin, and the $s$ axis, and let $u$ be a subparabolic or parabolic function depending only on the space variables, so that, considered as a function on the $N$ sphere, it is a subharmonic or harmonic function $u_{0}$. We take as a set which scans $D$ the set of points on the $s$ axis with non-negative integer ordinate values. In accordance with our criteria for the use of unbounded nested domains in Theorems 5.4 and 5.5, we can choose as $D_{n}$ the direct product of the $N$ sphere of radius $1-1 /(n+1)$, center the origin, and the $s$ axis. It is now clear that it suffices to check the conditions of Theorems 5.4 and 5.5 at a single point of our set which scans the domain $D$, and we choose the origin. In terms of $u_{0}$, the condition in Theorem 5.4 that Theorem 5.1 be applicable becomes the condition that $\left|u_{0}\right|$ have an integral, with respect to ordinary surface area on the boundary of the $N$ spheres with center the origin and radius less than 1 , which defines a bounded function of the radius. This is the well-known condition sufficient that $u_{0}$ have a boundary limit along almost every radial path to the boundary. Theorem 5.1 asserts that, under this hypothesis, and also under hypothesis $H_{u}$ which we shall show below is always satisfied, $u_{0}$ has a boundary limit along almost every $N$ dimensional Brownian. motion from the center to the boundary. The condition of Theorem 5.5 that $u$ be a stochastically ramified Dirichlet solution becomes, in terms of $u_{0}$, the well-known condition that $u_{0}$ be uniformly integrable on the surfaces of the $N$ spheres with center the 
origin and radius less than 1 . This condition is known to be necessary and sufficient that $u_{0}$ be representable by the Poisson integral, with a Lebesgue integrable boundary function. Parabolic measure on the boundary of $D$ reduces to harmonic measure (determined by the Poisson kernel as density function), so that this representation of $u_{0}$ corresponds to the parabolic average of an ordinary single valued function on the boundary of $D$. Thus, in this simple special case, ordinary Dirichlet solutions and stochastically ramified solutions are the same, as would be expected in view of the simple nature of $D$. Further details on this case from the probability point of view will be found in [2]. The present approach shows how the elegant classical results on subharmonic and harmonic functions defined on a sphere can be extended to the most general subparabolic and parabolic functions defined on an arbitrary open set, simply by choosing the paths of approach to the boundary carefully, and making the corresponding definition of a boundary function.

For a second example, set $N=1$, and consider a function $u$, non-negative and parabolic on the upper half-plane. Then $u$ has a boundary limit along almost every Brownian trajectory from a point in the upper half-plane to the axis of abscissas. It is interesting to identify the limiting value. According to a theorem of Widder [7], $u$ is given by

$$
u(\xi, s)=\int_{-\infty}^{\infty} \frac{\exp \left(-(\xi-\eta)^{2} / 4 s\right)}{2(\pi s)^{1 / 2}} d \psi(\eta),
$$

where $\psi$ is monotone nondecreasing, and the integral converges for all $s>0$. It is easy to see that almost all trajectories from any point of the upper halfplane to the axis of abscissas meet this line in such a way that they intersect every ray from the point of meeting in to the upper half-plane arbitrarily near the point of meeting. Since $\lim _{t \downarrow 0} u(\xi, s)=\psi^{\prime}(\xi)$ whenever the latter derivative exists, it follows that the boundary limit along a Brownian trajectory is almost always the value of $\psi^{\prime}$ at the boundary point. If $u$ is a parabolic function on the upper half-plane, and if $u$ is dominated by a positive parabolic function, $v$, the representation $u=v-(v-u)$ shows that $u$ can be written as the difference between two positive parabolic functions, and thus has the form (5.8) with $\psi$ the difference between two monotone nondecreasing functions. The integrand in (5.8) is the density determining the parabolic measure relative to $D$ at the point $(\xi, s)$. Hence, $u$ is the parabolic average of its boundary function, that is, $u$ is a stochastically ramified Dirichlet solution, if and only if $\psi$ is absolutely continuous on every compact interval. Thus the stochastically ramified and ordinary Dirichlet solutions coincide, and are determined by (5.8) with $d \psi(\eta)$ replaced by $\psi^{\prime}(\eta) d \eta$.

As a last remark in this general area, we observe that Theorem 5.1 remains true if the initial point $z$ is an irregular boundary point of $D$, or has an initial distribution confined to the interior and the irregular boundary points of $D$, because this case is reduced at once to single initial points in $D$. 
6. Local continuity of subparabolic functions and the fine topology. Let $D$ be a finite open set of $N+1$ space, and let $z_{0}$ be either an interior point or an irregular boundary point of $D$. Then, if $u$ is a function defined on $D$, we shall say that $u$ has a limit at $z_{0}$ on almost every Brownian trajectory from $z_{0}$ if, whenever $\{z(t), 0 \leqq t \leqq \infty\}$ is a Brownian trajectory process with initial point $z_{0}, \lim _{t \rightarrow 0} u[z(t)]$ exists with probability 1 . In general, it is not necessarily true that, for a specified Brownian trajectory process, the probability that this limit exists is well defined, or that, if the limit exists with probability 1 for a specified Brownian trajectory process, it will exist with probability 1 for a second specified Brownian trajectory process. However we shall deal only with semicontinuous and continuous functions, for which these matters have easy answers. If $u$ is continuous on $D$, it is clear that there is no problem. If $u$ is semicontinuous, it is the limit of a monotone sequence of continuous functions, and the problem is trivially reduced to the continuous case. In both cases, the probability that the above limit exists is well defined, and independent of the specified Brownian trajectory process. In both cases, as is shown by an application of the usual zero-one law reasoning to processes with independent increments, for a given Brownian trajectory process, the limit exists with probability 1 if it exists with positive probability, and if so is a finite or infinite constant with probability 1 . The constant is independent of the Brownian trajectory process involved. If the initial point $z_{0}$ is no longer fixed, but is given an initial distribution on $D$ and its irregular boundary points, no change in the above remarks is needed except that the limit need no longer be a constant.

We shall use the above remarks when $u$ is subparabolic. They would be quite superfluous if condition $H_{u}$ were satisfied, since then all the stochastic processes involved would be separable, but we note that we have not proved separability, and in fact, even assuming that $D$ is $N+1$ space itself, so that $u[z(t)]$ is always well defined, our remarks do not imply, directly at least, that the $u[z(t)]$ process is separable.

The following theorem will be used in proving the universal validity of condition $H_{u}$, and thereby eliminating these measurability problems.

Theorem 6.1. Let $u$ be a function subparabolic on a finite open set $D$, and let $z_{0} \in D$. Then $u$ has the limit $u\left(z_{0}\right)$ on almost every Brownian trajectory from $z_{0}$.

Since $u$ is upper semicontinuous, the result is trivial unless $u\left(z_{0}\right)$ is finite, as we shall suppose in the following. Applying Lemma 4.2, it is no restriction to assume that $D$ is actually $N+1$ space, and that $|u(z)|$ is small for large $|z|$, and we shall do so. Hence, by Theorem 4.3 , if $\{z(t), 0 \leqq t \leqq \infty\}$ is a Brownian trajectory process with initial point $z_{0}$, the process $\{u[z(t)], 0 \leqq t \leqq 1\}$ is a semimartingale. According to a standard semimartingale theorem [1], if $S$ is any denumerable set of real numbers,

$$
\lim _{t \rightarrow 0, t \in S} u[z(t)]=y
$$


exists with probability 1 , and has a finite expectation, with

$$
E\{y\}=\lim _{t \rightarrow 0} E\{u[z(t)]\} \geqq E\{u[z(0)]\}=u\left(z_{0}\right) .
$$

On the other hand, $y \leqq u\left(z_{0}\right)$, since $u$ is upper semicontinuous. Hence

$$
\lim _{t \rightarrow 0, t \in S} u[z(t)]=u\left(z_{0}\right)
$$

with probability 1 . The unfortunate presence of $S$ is due to the fact that we have not yet proved the universal validity of condition $H_{u}$. Since $u$ is upper semicontinuous,

$$
\limsup _{t \downarrow 0} u[z(t)] \leqq u\left(z_{0}\right),
$$

and $u$ is upper semicontinuous, as a function of the parameter, on every continuous Brownian trajectory. Combining these facts with (6.1), we find that $u$ must have the limit $u\left(z_{0}\right)$ at $z_{0}$ along almost every Brownian trajectory from $z_{0}$, as was to be proved.

This theorem implies that, if $u$ is subparabolic on a finite open set $D$, and if $\{z(t), 0 \leqq t \leqq \infty\}$ is a Brownian trajectory process with an arbitrary distribution of initial points, then, if $t_{0} \geqq 0$,

$$
\lim _{t \downarrow z_{0}} u[z(t)]=u\left[z\left(t_{0}\right)\right]
$$

when the value of $z\left(t_{0}\right)$ lies in $D$, with probability 1 . This result is slightly stronger than the theorem, but it by no means implies that condition $H_{u}$ is satisfied.

The following rather obvious lemma will be used in the proof of the next theorem. Its proof will be included, since it is less obvious than its truth.

Lemma 6.2. Let $D$ be a finite open set, and let $z_{0}$ be an irregular boundary point of $D$. Then there is a bounded open subset $D_{0}$ of $D$, such that $z_{0}$ is an irregular boundary point of $D_{0}$, but that all other boundary points of $D_{0}$ are in $D$.

We can and shall suppose that $D$ is bounded. If it is not, we can replace it by the set of its points at distance less than 1 from $z_{0}$. For each positive integer $n$ choose $\delta_{n}$ so small, although positive, that $\delta_{n}<\delta_{n-1}$, that $\delta_{n}<1 / n$, and that the probability that a Brownian trajectory from $z_{0}$ will meet the complement of $D$ less $z_{0}$, in time at most $\delta_{n}$, is at most $2^{-n}$. Let $D_{n m}$ be the set of those points of $D$ with $\operatorname{ord}\left(z_{0}\right)-\delta_{n}<\operatorname{ord}(z)$, or else with

$$
\operatorname{ord}\left(z_{0}\right)-\delta_{n-1}<\operatorname{ord}(z) \leqq \operatorname{ord}\left(z_{0}\right)-\delta_{n}
$$

and at distance greater than $1 / m$ from the boundary. Then $D_{n m}$ is open, and the probability that a Brownian trajectory from $z_{0}$ meets the complement of $D_{n m}$, less $z_{0}$, between times 0 and $\delta_{n}$ inclusive can be made at most $2^{-n+1}$ by choosing $m$ large. For such a choice of $m$, set $D_{n m}=D_{n}$. Then $D_{0}=\bigcap_{1}^{\infty} D_{n}$ 
fulfills the assertions of the lemma. If desired, we could modify the construction to obtain $D_{0}$ in such a way that all its boundary points other than $z_{0}$ are regular.

The next theorem is a generalization of the preceding one in that $z_{0}$ is now merely an irregular boundary point of $D$, rather than an interior point. We were helped in proving the preceding theorem by the upper semicontinuity of $u$ at $z_{0}$. This of course will no longer be available, since $u$ will not even be defined at that point. To compensate, we shall add hypothesis $H_{u}$, whose universal validity will be proved in the next section.

THeOREM 6.3. Let $z_{0}$ be an irregular boundary point of a finite open set $D$. Let $u$ be a function subparabolic, satisfying hypothesis $\mathrm{H}_{u}$, and bounded from above on $D$. Then $u$ has a (constant) limit $c,-\infty \leqq c<\infty$, at $z_{0}$ along almost all Brownian trajectories from $z_{0}$.

In view of the preceding lemma, we can and shall suppose that $D$ is bounded, and is a subset of a larger open set on which $u$ is defined and satisfies the hypotheses of the theorem, and which contains every boundary point of $D$ other than $z_{0}$. Suppose at first that $u$ is bounded from below. Let $\left\{z^{*}(t)\right.$, $0 \leqq t \leqq \infty\}$ be a Brownian trajectory process, with initial point $z_{0}$, stopped on the boundary of $D$, that is, when the trajectories reach the boundary for the first time after the initial point. Then, computing conditional expectations just as we have done above, we find that, if $0<s<t$,

$$
u\left[z^{*}(s)\right] \leqq E\left\{u\left[z^{*}(t)\right] \mid z(r), r \leqq s\right\} .
$$

In fact the inequality is trivial after the trajectories have stopped, and before stopping we can apply Theorem 4.4 . Hence the process $\left\{u\left[z^{*}(t)\right], 0<t \leqq \infty\right\}$ is a semimartingale, a separable semimartingale because condition $H_{u}$ is satisfied. According to a standard semimartingale theorem, it follows that $\lim _{t \rightarrow 0} u\left[z^{*}(t)\right]$ exists with probability 1 . The theorem is thus proved, under the added hypothesis that $u$ is bounded from below. Without this hypothesis, the result can be applied to $\max [u, n]$ for all $n$, to prove the theorem. We have already remarked that the limit is, with probability 1 , a constant. Note that the use of condition $H_{u}$ has made the proof of this theorem easier than that of the less general Theorem 6.1. This condition could not be made one of the hypotheses of the latter theorem because that theorem will be used to prove the universal validity of the condition.

By a fine neighborhood of the finite point $z_{0}$ in $N+1$ space we shall mean any set containing the points satisfying the inequalities

$$
u(z)>u\left(z_{0}\right)-\epsilon, \quad\left|z-z_{0}\right|<\epsilon,
$$

for some function $u$, subparabolic on an ordinary neighborhood of $z_{0}$, and some positive number $\epsilon$, or any intersection of finitely many of these sets. Note that these inequalities define an $F_{\sigma}$ set in a neighborhood of $z_{0}$ on which $u$ is defined. The neighborhood system defined in this way determines a Hausdorff 
topology, which we shall call the fine topology, following Cartan's terminology in the case relevant to subharmonic functions, that is, to Laplace's equation rather than to the heat equation. The fine topology is that with the smallest collection of open sets needed to make all subparabolic functions continuous. A point set will be called thin at a finite point if that point is not a limit point of the set in the fine topology.

If $u(z)$ is defined as -1 above $z_{0}$ and 0 otherwise, we find that the halfspace determined by the inequality ord $(z) \leqq \operatorname{ord}\left(z_{0}\right)$ is a fine neighborhood of $z_{0}$. Thus we could add the inequality $\operatorname{ord}(z) \leqq \operatorname{ord}\left(z_{0}\right)$ to those in (6.4) without changing the topology. An only slightly more complicated example shows that we can even add the inequality $\operatorname{ord}(z)<\operatorname{ord}\left(z_{0}\right)$ to those in (6.4) without changing the topology, if only we also add the condition that the point $z_{0}$ itself lie in every neighborhood of $z_{0}$. In other words the set where $\operatorname{ord}(z)$ $\geqq \operatorname{ord}\left(z_{0}\right)$ is thin at $z_{0}$.

Since, according to Theorem 6.1, $u$ in (6.4) has the limit $u\left(z_{0}\right)$ at $z_{0}$ along almost all Brownian trajectories with initial point $z_{0}$, it follows that, given any fine neighborhood of a point, almost every Brownian trajectory of a given process from the point lies in that neighborhood for an initial parameter interval whose length may depend on the trajectory. We observe that this statement is not the same as the statement that almost all Brownian trajectories of a specified process with specified initial point are continuous at the parameter value 0 in the fine topology.

A point set $A$ will be called stochastically thin at a finite point $z_{0}$ if almost every Brownian trajectory, of any process with initial point $z_{0}$, has an initial $\operatorname{arc}$ (not counting $z_{0}$ itself) which lies in the complement of $A$. The parameter interval yielding this arc may depend on the trajectory. It is clear that, if $A$ is compact, or an $F_{\sigma}$ set, it is sufficient to prove that the trajectories of a single Brownian trajectory process have this property. We have proved (Theorem 3.1) that, if $A$ is the complement of an open set $D$, then a finite boundary point of $A$ is irregular for the Dirichlet problem (heat equation) on $D$ if and only if $A$ is stochastically thin at the point. We note that this is not the same as prescribing that the boundary be stochastically thin at the point. For example, if $N=1$, the origin is a regular boundary point of the upper halfplane, but the boundary is thin at the origin.

Theorem 6.4. If a set $A$ is thin at $z_{0}, A$ is stochastically thin at $z_{0}$. Conversely, if $A$ is an $F_{\sigma}$ set, stochastically thin at $z_{0}$, then $A$ is thin at $z_{0}$.

The fact that, if $A$ is thin at $z_{0}$, it is stochastically thin there, is merely a reformulation of the fact that, given any fine neighborhood of $z_{0}$, almost every Brownian trajectory of a specified process from that point lies in that neighborhood for an initial parameter interval. Conversely, suppose that $A=\cup_{1}^{\infty} A_{n}$, where $A_{n}$ is compact, and that $A$ is stochastically thin at $z_{0}$. It is no restriction in proving that $A$ is thin at $z_{0}$ to assume that every point of $A$ has ord value less than that of $z_{0}$, and we shall assume this to be true. Cutting off every 
$A_{n}$, and thereby $A$, below some horizontal hyperplane, the same for all $n$, below $z_{0}$, but close to $z_{0}$, we can assume that $u_{A}\left(z_{0}\right)<1 / 4$. Let $B_{1}$ be an open set containing $A_{1}$, with compact closure $\bar{B}_{1}$, so small that, if $C_{1}=A \cup \bar{B}_{1}$,

$$
u_{C_{1}}\left(z_{0}\right)<\frac{1}{4}+\frac{1}{3} .
$$

If $B_{1}, C_{1}, \cdots, B_{n}, C_{n}$ have been chosen, let $B_{n+1}$ be an open set, containing $A_{n+1} \cup C_{n}$, with compact closure $\bar{B}_{n+1}$, so small that, if $C_{n+1}=A \cup \bar{B}_{n+1}$,

$$
u_{C_{n+1}}\left(z_{0}\right)<u_{C_{n}}\left(z_{0}\right)+3^{-n-1} \text {. }
$$

Then

$$
A \subset C_{1} \subset B_{2} \subset C_{2} \subset B_{3} \subset \cdots
$$

and, if $B=\cup_{1}^{\infty} B_{n}=\cup_{1}^{\infty} C_{n}$, it follows that $B$ is open and $B \supset A$. Now, by our construction,

$$
\begin{aligned}
& u_{C_{n}}\left(z_{0}\right)<\frac{1}{4}+\frac{1}{3}+\cdots+\frac{1}{3^{n}}<\frac{3}{4}, \\
& u_{B}\left(z_{0}\right)=\lim _{n \rightarrow \infty} u_{C_{n}}\left(z_{0}\right) \leqq \frac{3}{4} .
\end{aligned}
$$

Hence $u_{B}$ is a superparabolic function, with value at most $3 / 4$ at $z_{0}$, and, since $B$ is open, with value 1 on $B$. Since $-u_{B}$ is subparabolic, the inequalities

$$
-u_{B}(z)>-u_{B}\left(z_{0}\right)-1 / 4, \quad\left|z-z_{0}\right|<1,
$$

define a fine neighborhood of $z_{0}$ which contains no point of $B$, and hence no point of $A$. Thus $A$ is thin at $z_{0}$, as was to be proved.

Theorem 6.3 can now be rephrased very simply, in terms of the fine topology and associated concepts. That theorem states that a subparabolic function $u$ which is bounded from above (satisfying the condition $H_{u}$ which we shall show in $\$ 7$ is satisfied by every subparabolic function) has a finite or negatively infinite limit at every irregular boundary point of its domain, in terms of approach to that boundary point in the fine topology. To see this, we need only remark that, if $c$ has the significance given to it in Theorem 6.3, and if $c$ is finite, then the $z$ set defined by the inequality $|u(z)-c|>\epsilon$ is an $F_{\sigma}$ set which is stochastically thin at $z_{0}$, if $\epsilon>0$, and is therefore thin at $z_{0}$. In other words, $u$ is arbitrarily near $c$ in a sufficiently small fine neighborhood of $z_{0}$. The case of an infinite limit is treated similarly. The limit has no simple evaluation, in general. However, it is almost trivial that Theorem 5.3, with $u$ bounded from above, can be extended to allow the initial point $z$ to be an irregular boundary point in that theorem, with $u(z)$ defined as the fine limit which we have just proved exists there. Then in this case $c$ is at most the parabolic average, in the obvious limiting sense, relative to $D$ of $u$ at $z$. In particu- 
lar, if $u$ is a Dirichlet solution, there is equality, and in this case the fine limit at the irregular point must be finite. The corresponding result for subharmonic and harmonic functions is well known.

7. The continuity properties of subparabolic functions, in the large. In this section we shall discuss the continuity properties of subparabolic functions along Brownian trajectories, as functions of the parameter. From another point of view, this is a discussion of the way in which Brownian trajectories pass through the discontinuities of such functions.

Theorem 7.1. Let $u$ be a function subparabolic on a finite open set D. Then, if $z_{0}$ is a finite point, almost no Brownian trajectory from $z_{0}$ passes through an infinity of $u$ (neglecting $z_{0}$ itself, if $u\left(z_{0}\right)=-\infty$ ).

In view of Lemma 4.2, we can and shall assume in proving this theorem that $D$ is finite $N+1$ space, and that $u$ is near 0 for large $|z|$. Then, according to Theorem 4.3 , if $\{z(t), 0 \leqq t \leqq \infty\}$ is a Brownian trajectory process with initial point $z_{0}$, the process $\{u[z(t)], 0 \leqq t<\infty\}$ is a semimartingale, except that we exclude 0 from the parameter set if $u\left(z_{0}\right)=-\infty$. Now the sample functions of any semimartingale with parameter set a compact interval are almost all bounded on the set of rational points of the interval. Since $u$ is continuous, in the obvious extended sense, whenever it has the value $-\infty$, a sample function of the $u[z(t)]$ process cannot be bounded on the set of rational points of a compact parameter interval if the corresponding Brownian trajectory passes through an infinity of $u$ at any point of the interval. Hence the theorem is true.

This theorem gives some idea of how rare the infinities of a subparabolic function are, or, from another point of view, of how much of $N+1$ space a Brownian trajectory traverses. For example, if $u$ is a function of the space variables alone, this theorem implies that almost no $N$ dimensional Brownian motion from a point will pass through a specified set of zero capacity (see [2]).

THEOREM 7.2. If $D$ is a finite open set, and if $u$ is subparabolic on $D$, then condition $H_{u}$ is satisfied.

Applying Lemma 4.2, we can and shall assume that $D$ is finite $N+1$ space, in proving this theorem. Let $\{z(t), 0 \leqq t \leqq \infty\}$ be a Brownian trajectory process with initial point $z_{0}$. We can assume that $u\left(z_{0}\right)>-\infty$, and we shall prove that $u$ is finite valued and right continuous as a function of the parameter on almost every trajectory of this process. Let $\epsilon$ be a positive number, and let $\tau_{1}(\omega)$ be the infimum of the values of $t$ for which

$$
\sup _{0 \leqq s \leqq t}\left|u[z(s, \omega)]-u\left(z_{0}\right)\right| \geqq \epsilon .
$$

To see that $\tau_{1}$ is a measurable function, we note first that the inequality $\tau_{1}(\omega)>t$ defines exactly the $\omega$ set not satisfying (7.1) for fixed $t$. Hence all we 
need verify is that the left side of (7.1), for fixed $t$, defines a measurable $\omega$ function $y$. This assertion is obviously true if $u$ is continuous, because we can then restrict $s$ to be rational, so that $y$ is merely the superior limit of a sequence of random variables. In general, $u$ is the limit of a monotone decreasing sequence $\left\{u_{n}\right\}$ of continuous functions, and $y$ is then the limit of the corresponding random variables, with $u[z(t)]$ replaced by $u_{n}[z(t)]$. We note for later use that the argument would still be valid if $z_{0}$ had an initial distribution instead of being a constant. This argument proves also that, for any $t>0$, the inequality $\tau_{1}(\omega)>t$ defines an $\omega$ set depending only on $z(s)$ for $s \leqq t$. Hence trajectories for parameter values of the form $\tau_{1}+t, t \geqq 0$, behave like Brownian trajectories of a process starting from time 0 with an initial distribution, that of $z\left(\tau_{1}\right)$. The function $z\left(\tau_{1}\right)$ is itself a measurable $\omega$ function because it is the limit, as $n \rightarrow \infty$, of the sequence $\left\{z_{n}\right\}$ of random variables defined by

$$
z_{n}(\omega)=z\left(\frac{j}{n}, \omega\right) \quad \text { if } \frac{j-1}{n} \leqq \tau_{1}(\omega)<\frac{j}{n} .
$$

According to Theorem 7.1, $\tau_{1}(\omega)>0$ with probability 1 , because $u$ has the limit $u\left(z_{0}\right)$ along almost every trajectory from $z_{0}$. Applying this theorem, with an initial distribution of trajectories this time, we find that, if $\tau_{1}(\omega)<\infty$, $u\left[z\left(\tau_{1}+t\right)\right]$ defines a function of $t$ which is right continuous when $t=0$, with probability 1 . We then define $\tau_{2}^{\prime}$ as 0 if $\tau_{1}$ has the value $+\infty$, and otherwise just like $\tau_{1}$ except that $u\left[z\left(\tau_{1}+t\right)\right]$ replaces $u[z(t)]$ and $z\left(\tau_{1}\right)$ replaces $z_{0}$, and define $\tau_{2}=\tau_{1}+\tau_{2}^{\prime}$. We then continue in the same way, defining a sequence $\tau_{1} \leqq \tau_{2} \leqq \cdots$, where equality can hold only with probability 0 except that, if $\tau_{j}(\omega)=\infty$, then $\tau_{j+1}(\omega)=\cdots=\infty$ also. If $\beta$ is any transfinite ordinal of the second class, we can define $\tau_{\beta}$ by induction as follows. If $\tau_{\alpha}$ has already been defined for $\alpha<\beta$, define $\tau_{\beta}$ as $\sup _{\alpha<\beta} \tau_{\alpha}$, if $\beta$ is a limit ordinal. If $\beta$ is not a limit ordinal, go from $\tau_{\beta-1}$ to $\tau_{\beta}$ in exactly the same way as from $\tau_{j-1}$ to $\tau_{j}$ above. Thus $\tau_{\beta}$ is defined by induction, and it is clear that, if $\alpha<\beta$, then $\tau_{\alpha}<\tau_{\beta}$ with positive probability unless both random variables are equal to $+\infty$ with probability 1 . Hence $E\left\{\arctan \tau_{\alpha}\right\}$ increases with $\alpha$ to the value $\pi / 2$, that is, $\tau_{\beta}=+\infty$ for some ordinal number $\beta$ of the second class. Now this means, if we delete the exceptional $\omega$ sets of measure 0 which arise at each of the at most enumerably many steps to the $\beta$ th, that $u$, as a function of the trajectory parameter, has right hand oscillation at most $2 \epsilon$ on the remaining trajectories. Since $\epsilon$ is arbitrary here, the theorem is proved.

In this paper we have repeatedly discussed stochastic processes of the form $\{u[z(t)], t \in T\}$, where $T$ is some interval, $u$ is a function defined on a set of $N+1$ space, and the $z(t)$ process is a process whose trajectories lie in the domain of definition of $u$. In the study of continuous parameter stochastic processes, there are well-known measure difficulties, caused, among other things, by the fact that certain desirable operations may lead to nonmeasura- 
ble functions on the basic measure space. One standard tool in dealing with these difficulties is, for each parameter value, to change the corresponding random variable of the process, replacing it by one equal to it almost everywhere. This theoretical flexibility, which leaves unchanged the joint finite dimensional distributions of the random variables of the process, is illusory in the present case of the $u[z(t)]$ process, because $u$ is specified, and cannot be changed, and the $z(t)$ process is prescribed to have almost all its sample functions continuous, so that no significant changes in each $z(t)$ can be made. We have thus been unable to assume that the $u[z(t)]$ process is, or at least can be made, separable, because the above type of change has been unavailable. Now that it has been shown that condition $H_{u}$ is always satisfied, however, the $u[z(t)]$ process is recognized to be necessarily (trivially) separable, and even separable relative to the closed sets.

We can now complete our analysis of the character of a subparabolic function on Brownian trajectories. We have proved that $u$ is right continuous as a function of the trajectory parameter, on almost every trajectory, while the trajectory is in the domain of the function. We can go further than this. In view of the connection we have established between subparabolic functions and semimartingales, and of the fact that almost all sample functions of a separable semimartingale have finite one-sided limits at all points, it follows that a subparabolic function has a finite left-sided limit at each point of almost every trajectory, in terms of the trajectory parameter. Since the function is upper semicontinuous, this limit is less than or equal to the value of the function at the discontinuity. In other words, aside from the possible infinity of the function at the initial point of the trajectory, the discontinuities of the function on almost every trajectory are upward going jumps at which there is right continuity.

We now carry further the discussion started at the end of $\$ 4$, and generalized somewhat in $\S 5$, on the stochastic process $\left\{u\left[z\left(\tau_{t}\right)\right], t \in T\right\}$. Here $u$ is subparabolic on a finite open set $D, D_{t}$ is an open subset of $D$, which does not decrease when $t$ increases, the $z(s)$ process is a Brownian trajectory process with initial point $z_{0}$, a specified point of $D$ and included in every $D_{t}$. It is supposed that either (a) in each $D_{t}, u$ is dominated from above by a stochastically ramified Dirichlet solution, or (b) $u$ is itself a stochastically ramified Dirichlet solution. We have seen in $\S 5$ that in this work $D_{t}$ may have boundary points in common with $D$, if $u$ is interpreted properly at such points. Then the $u\left[z\left(\tau_{t}\right)\right]$ process is a semimartingale, under (a), a martingale under (b). Now suppose that (c) if $s \in T, \lim _{t \downarrow s} \tau_{t}=\tau_{\varepsilon}$ with probability 1 . This hypothesis will be satisfied if, for each $s, \bigcap_{s>t} D_{s}^{\prime}=D_{t}^{\prime}$, where $D_{t}^{\prime}$ is the finite closure of $D_{t}$, and if the set of irregular boundary points of $D_{t}$, has parabolic measure 0 , relative to $D_{s}$, at $z_{0}$. Under (c), the sample functions of the $u\left[z\left(\tau_{t}\right)\right]$ process are almost all right continuous, and the process is therefore separable, so that almost all sample functions also have finite left sided limits at all parameter points. As we have remarked in $\$ 4$, it is always possible in this work, if 
$u\left(z_{0}\right)>-\infty$, to suppose that $T$ has a first point $t_{0}$, if we set $\tau_{t_{0}}=0$. Condition (c) will then be satisfied when $s=t_{0}$ if $\bigcap_{\bullet>t_{0}} D_{s}^{\prime}$ is the point $z_{0}$, that is, if the diameter of $D_{s}$ goes to zero with $s-t_{0}$. Under condition (a), we have seen that $E\left\{u\left[z\left(\tau_{t}\right)\right]\right\}$ defines a nonincreasing function of $t$ in accordance with the principle that the parabolic average of a subparabolic function, relative to an open set, at a specified point of the set, does not decrease when the set increases. Under condition (b) this expectation does not depend on $t$. Finally, under conditions (a) and (c) it follows from general semimartingale theory that this expectation function is right continuous, like the sample functions.

8. Potential theory. It would be presumptuous as well as foolhardy for the author to set up a theory of heat potential in any detail. However, since such a theory does not appear to have been presented from a real variable point of view, and since it will clarify considerably the results of this paper, and their relation to the corresponding results for classical potentials, the author is venturing where experts have not deigned to tread. There will be no attempt at completeness, but there will be an attempt to set up the basic definitions and to sketch the proofs of the basic theorems, in greatest generality. The nonprobabilistic aspects of the results obtained in this section are all familiar, in one form or another, in the special case of classical potentials. The latter case is obtained by choosing all domains of definition of functions to be unions of lines parallel to the $s$ axis, all distributions to be invariant under translations parallel to this axis, and all functions to be functions of the space variables only. The probability methods yield more detailed results than are known even in the classical case, however. The fact that all potentials have (stochastic) boundary functions, and that there is a simple characterization of (stochastically ramified) Dirichlet solutions, makes the analysis of potentials on the most general open sets no more difficult than their analysis on simple domains like rectangles or spheres, and makes the $N+1$ dimensional heat potential no more difficult than the $N$ dimensional Newtonian potential. In fact, if one wished to treat the latter independently, instead of treating it as a special case of the heat potential, all one would need to do below would be to replace $N+1$ space by $N$ space, make the obvious change of kernel, and interpret "Brownian trajectory" as "sample function of an $N$ dimensional Brownian motion."

We have already defined the heat potential of a distribution of positive and negative heat sources over $N+1$ space. The generating kernel was $\phi$, defined by (1.3). It is important to define a more general heat potential, that generated by a Green's function. Let $D$ be any finite open set of $N+1$ space. We wish to define potentials relative to $D$. This is done by first defining the Green's function of $D$. Let $\phi$ be defined by (1.3). If $z_{0}$ is a point of $D, \phi\left(z-z_{0}\right)$ defines a function on the boundary $C$ of $D$. This function obviously belongs to the class $L(D)$, since it is bounded above $z_{0}$ and vanishes elsewhere. Hence there is a corresponding Dirichlet solution for the heat equation on $D$, and the Green's function value $u\left(z_{0}, z\right)$ is defined as $\phi\left(z-z_{0}\right)$ less this solution 
at $z$. The Green's function, for fixed $z_{0}$, is therefore a function defined and superparabolic on $D$, parabolic except at the pole $z_{0}$, and has the boundary limit 0 at all regular boundary points. It has the stochastic boundary function 0 , and, according to Theorem 5.3, is non-negative. In particular, if $D$ is finite $N+1$ space, the Green's function value with pole $z_{0}$ reduces to $\phi\left(z-z_{0}\right)$ at $z$. The definition of potentials relative to $D$ is now given just as in the special case when $D$ is the whole space, first for finite valued heat source distributions confined to compact subsets of $D$, using the Green's function as the kernel, and then for heat source distributions which are finite on compact subsets, but whose value on noncompact subsets of $D$ may be infinite. The potential of a non-negative distribution is superparabolic, parabolic on open sets containing no heat sources.

In the following, we shall frequently deal with non-negative superparabolic functions. We observe that the negative of such a function is subparabolic and dominated from above by a positive stochastically ramified Dirichlet solution, the function 1 . Hence several theorems of $\$ 5$ are applicable. In particular, such a function has a stochastic boundary function. The following decomposition theorem is trivial, or nearly so, in the light of our previous results. Its significance will be seen later.

THEOREм 8.1. Let $u$ be a superparabolic and non-negative function on the finite open set $D$. Let $u_{2}$ be the stochastically ramified Dirichlet solution with the same stochastic boundary. function as $u$, and let $u_{1}=u-u_{2}$. Then $u_{1}$ is nonnegative, superparabolic and has the stochastic boundary function 0 . Conversely, if $u=u_{1}^{\prime}+u_{2}^{\prime}$ on $D$, where $u_{1}^{\prime}$ is superparabolic, with stochastic boundary function $0, u_{2}^{\prime}$ is parabolic, and both functions are non-negative, then $u_{1}^{\prime} \leqq u_{1}$, $u_{2}^{\prime} \geqq u_{2}$.

Only the converse is now nontrivial. To prove the converse, we need only remark that $u_{2}-u_{2}^{\prime}$ is parabolic, dominated from above by the positive stochastically ramified Dirichlet solution $u_{2}+1$, and has the stochastic boundary function 0 . Hence $u_{2}-u_{2}^{\prime} \leqq 0$, by Theorem 5.3 .

Let $\left\{D_{n}\right\}$ be a nested sequence of open subsets of the finite open set $D$, with union $D$, and define $z_{z}(t), \tau_{n}$ as in $\S 5$. We make no hypothesis on the compactness of the closure of $D_{n}$, however. Let $u$ be a function defined on $D$, with stochastic boundary function $f$. Then we shall say that $u$ has the boundary function $f$ in the $L_{1}$ sense if, for every choice of initial point $z$ of Brownian trajectories, and every choice of the nested sequence of open sets,

$$
E\{|f(z)|\}<\infty, \quad E\left\{\left|u\left[z_{z}\left(\tau_{n}\right)\right]\right|\right\}<\infty,
$$

for sufficiently large $n$, and

$$
\lim _{n \rightarrow \infty} E\left\{\left|u\left[z_{z}\left(\tau_{n}\right)\right]-f(z)\right|\right\}=0 .
$$

We stress that it is part of the condition that $u$ have a stochastic boundary 
function in the sense already used. In other words, there must be pointwise convergence as well as convergence in the mean. If $u$ is a stochastically ramified Dirichlet solution, $u$ has a boundary function in the $L_{1}$ sense, because of the uniform integrability condition of Theorem 5.5. In fact, uniform integrability of a sequence, known to converge with probability 1 , is necessary and sufficient for convergence in the mean of order 1 . Hence a parabolic function is a stochastically ramified Dirichlet solution if and only if it has a stochastic boundary function in the $L_{1}$ sense. In particular, this means that a parabolic function with the stochastic boundary function 0 in the $L_{1}$ sense must vanish identically. Another consequence of these remarks is that, if $u$ has the stochastic boundary function $f$, and if $|u|$ is dominated from above by a stochastically ramified Dirichlet solution, $u$ has its boundary function in the $L_{1}$ sense. It is clear that if $u$ and $v$ are functions which have boundary functions in this sense, then any linear combination of $u$ and $v$ has this same property. It follows that, in Theorem 8.1 , if $u$ has its stochastic boundary function in the $L_{1}$ sense, the same is true of $u_{1}$, since $u_{2}$ is a stochastically ramified Dirichlet solution, and as such must have its boundary function in this sense.

THEOREM 8.2. If $u$ is the potential relative to $D$ of a distribution of heat sources on $D$, then $u$ has the stochastic boundary function 0 in the $L_{1}$ sense.

It is no restriction to suppose that $u$ is the potential of a distribution of positive heat sources, because the most general distribution can be expressed as the difference between two such distributions. Then $u$ has a stochastic boundary function, because $u$ is non-negative and superparabolic. Suppose first that the distribution generating $u$ is confined to a compact subset of $D$. Then, using our usual notation involving a nested sequence $\left\{D_{n}\right\}$ of open subsets of $D$, with union $D$, and supposing first that the closure of each $D_{n}$ is a compact subset of $D$, and considering values of $n$ so large that $z \in D_{n}$, and that $A \subset D_{n}$, it is clear that, for each $z \in D, u\left[z_{z}\left(\tau_{n}\right)\right]$ converges boundedly to 0 with probability 1 . Hence

$$
\lim _{n \rightarrow \infty} E\left\{u\left[z_{z}\left(\tau_{n}\right)\right]\right\}=0 .
$$

Now suppose that each $D_{n}$ is restricted as stated, but that $\mu$ is arbitrary. Then the expectations in the preceding equation do not increase when $n$ increases, so that we can write, for $N$ so large that $z \in D_{N}$,

$$
\lim _{n \rightarrow \infty} E\left\{u\left[z_{z}\left(\tau_{n}\right)\right]\right\} \leqq E\left\{u\left[z_{z}\left(\tau_{N}\right)\right]\right\} .
$$

We shall show that the limit on the left is 0 . We hold $N$ fast in the following argument. From what we have already shown, it is clear that the limit on the left is unchanged if we delete from $\mu$ the contribution to $\mu$ from any specified compact subset of $D$. Since this subset can be chosen so large that 
$u$ is uniformly small on $D_{N}$, the right side of (8.1) can be made arbitrarily small by such a change of $\mu$. Hence the left side must vanish. We have now proved that $u$ has the stochastic boundary function 0 in the $L_{1}$ sense, and to finish the proof of the theorem we need only prove that we can remove the restriction we have imposed above that each $D_{n}$ be a set whose closure is a compact subset of $D$. If this restriction is not imposed, let $D_{n}^{\prime}$ be the set of those points of $D_{n}$ at distance less than $n$ from the origin, and simultaneously at distance greater than $1 / n$ from the boundary of $D$. Then, if $\tau_{n}^{\prime}$ is the time a $z_{z}(t)$ process trajectory first meets the boundary of $D_{n}^{\prime}$, we have already proved that

$$
\lim _{n \rightarrow \infty} E\left\{u\left[z_{z}\left(\tau_{n}^{\prime}\right)\right]\right\}=0 .
$$

Here the sequence of expectations is monotone, because of the properties of parabolic averages relative to nested sets. In accordance with these same properties,

$$
E\left\{u\left[z_{z}\left(\tau_{n}\right)\right]\right\} \leqq E\left\{u\left[z\left(\tau_{n}^{\prime}\right)\right]\right\}
$$

and it follows that the sequence of the expectations on the left also has limit 0 , as was to be proved.

As would be expected, in view of classical potential theory, the converse of Theorem 8.2 is true.

THEOREM 8.3. Let $u$ be a non-negative superparabolic function on a finite open set $D$, and suppose that $u$ has the stochastic boundary function 0 in the $L_{1}$ sense. Then $u$ is the potential relative to $D$ of a non-negative distribution of heat sources.

To prove this theorem, we first remark that the Riesz decomposition theorem for classical potentials is valid for heat potentials, with no change in the principle of its proof. We shall use it in the following form: If $u$ is a nonnegative superparabolic function on a finite open set $D$, there is a positive distribution of heat sources on $D$, determining a potential $u_{1}$, such that $u_{2}=u-u_{1}$ is non-negative and parabolic on $D$. In our case, $u$ and $u_{1}$ have the stochastic boundary function 0 in the $L_{1}$ sense. Hence $u_{2}$ also has the stochastic boundary function 0 in this sense, so that, as we have remarked above, $u_{2}$ vanishes identically, and the proof of the theorem is complete.

The significance of Theorem 8.1 is now clear, if $u$ in that theorem has a stochastic boundary function in the $L_{1}$ sense. In that case the decomposition of that theorem is precisely the Riesz decomposition.

As an example of the theorem, we prove that the function $u_{A, D}$ discussed in Theorem 2.2 is a potential, relative to $D$, if $A$ is closed relative to $D$ in the unextended topology. According to Theorem 2.2, $u_{A ; D}$ is superparabolic on $D$, and has values between 0 and 1 inclusive. Moreover we remarked in $\$ 5$ that $u_{A, D}$ has the stochastic boundary function 0, if $A$ is as stated. More 
precisely, what was noted in $\$ 5$ was that $u_{A, D}$ has the limit 0 along almost every Brownian trajectory from a point of $D-A$ to the boundary of $D$, considering only the trajectories which meet the boundary of $D$ before passing through $A$. However this weaker assertion obviously implies the stronger one. Since $u_{A, D}$ is bounded, this function must have the boundary function 0 in the $L_{1}$ sense, and is therefore a potential. This potential is the equilibrium potential, relative to $D$, and is the direct generalization of the equilibrium (capacitary) potential in the Newtonian case. The equilibrium potential obtained here reduces to the latter one if $D$ and $A$ are chosen appropriately, to make superparabolic functions on $N+1$ space reduce to superharmonic functions on $N$ space and if distributions are chosen on $N+1$ space to be invariant under translations parallel to the $s$ axis, as discussed above.

Let $u$ be superparabolic and non-negative on a finite open set $D$, and suppose that $u$ has its stochastic boundary function in the $L_{1}$ sense. Then we have seen that we can write $u=u_{1}+u_{2}$, the Riesz decomposition, in which $u_{1}$ is a potential relative to $D$ and $u_{2}$ is a stochastic Dirichlet solution. It is easy to verify that this decomposition is unique. If $u$ has continuous second partial derivatives, an application of Green's theorem yields the distribution determining the potential $u_{1}$ explicitly in terms of $u$. This distribution is that determined by the density $-G u$, where $G$ is the operator defined by (1.2). Going on from this special case, one can conclude, as in the case of the classical potentials, that the distribution determining $u_{1}$ is always uniquely determined by $u$. It then follows that a potential on $D$ due to a (not necessarily positive) distribution of heat sources determines the corresponding distribution uniquely.

The sweeping out process of classical potential theory has the following generalization in the present theory, formulated at first in terms of transformations between superparabolic functions rather than between heat source distributions. The problem is complicated by the peculiar continuity properties of superparabolic functions, which compel us to use not the values of these functions on specified sets, but the limits of the functions along Brownian trajectories to the sets. Let $D$ be a finite open set of $N+1$ space. This set will be held fast throughout the following discussion, and will not be indicated in the notation. In the most important case, $D$ is the whole finite space. Let $v$ be a non-negative function, defined and superparabolic on $D$, with stochastic boundary function 0 . Let $A$ be a subset of $D$, closed in the unextended topology, and let $D^{\prime}=D-A$. Let $f$ be the stochastic boundary function of $v$, considered only on $D^{\prime}$. Then, according to Theorem 5.3,

$$
E\{f(z)\} \leqq v(z),
$$

$$
z \in D^{\prime}
$$

What we wish to do is to increase $v$, if necessary but if so by as little as possible, to make this function parabolic on $D^{\prime}$, leaving it superparabolic on $D$. The new function will be called $u_{A, v}$. If $z \in D^{\prime}$, we define $u_{A, v}(z)$ as the left side of (8.2). Thus $u_{A, v}$ on $D^{\prime}$ is a stochastically ramified Dirichlet solution, 
and, as such, is parabolic on $D^{\prime}$. Let $G_{n}$ be an open subset of $D$, and define $f_{n}$ $\left[g_{n}\right]$ as the stochastic boundary function of $v$, when $v$ is considered defined only on $D^{\prime} \cup G_{n}\left[G_{n}\right]$. Let $z$ be a point of $D$, and choose $G_{n}$ in such a way that $z \in G_{n}$, that $G_{1} \supset G_{2} \supset \cdots$, and that the diameter of $G_{n}$ approaches 0 with $1 / n$. Then, according to the discussion in previous sections,

$$
v(z) \geqq E\left\{g_{n}(z)\right\} \geqq E\left\{f_{n}(z)\right\},
$$

and these expectations do not decrease when $n$ increases. We define

$$
u_{A, v}(z)=\lim _{n \rightarrow \infty} E\left\{f_{n}(z)\right\} \text {. }
$$

This definition reduces to the previous one, for $z \in D^{\prime}$. We have seen in $\S 7$ that, if $f_{n}$ were replaced by $g_{n}$ in this definition, the limit in (8.4) would be $v(z)$. Thus $u_{A, v}(z)=v(z)$ if, for large $n$, almost every Brownian trajectory from $z$ meets the boundary of $G_{n}$ for the first time in a point of $A$. In particular, this equality will be true if $z$ is an interior point of $A$. More generally, it will be true if (a) for every $\epsilon>0$, the probability approaches 1 when $n \rightarrow \infty$ that a Brownian trajectory that first meets the boundary of $G_{n}$ in a point of $D^{\prime}$ will then meet $A$ in time $\leqq \epsilon$, and if (b) it is also known that the contribution to $E\left\{f_{n}(z)\right\}$ from an $\omega$ set of measure $\leqq \epsilon$ approaches 0 with $\epsilon$. These conditions are satisfied if $v$ is bounded and if $D^{\prime}$ is thin at $z$.

The procedure we have used to define $u_{A, v}$ is a generalization, or refinement, of that used to define $u_{A, D}$, and by choosing $v$ properly we can obtain $u_{A, D}$. In fact, it is sufficient to take $v$ as $u_{B, D}$, where $B$ is any set which contains $A$, is a subset of $D$, and is closed in the unextended topology. This fact is trivial if $B$ is chosen so that every point of $A$ is an inner point of $B$, because with this choice of $v$ the function $v$ will be identically 1 on $A$, and is easy to verify with any choice.

In the light of this analysis, it is not surprising that $u_{A, v}$ shares many of the properties of $u_{A, D}$. The function $u_{A, v}$ is, by definition, parabolic on $D^{\prime}$, and has the same stochastic boundary function on its boundary as $v$. Moreover, like $u_{A, D}, u_{A, v}$ is superparabolic on $D$. In fact the proof of this property of $u_{A, v}$ goes through with no real change in principle, although the formal justification is more sophisticated. For example, the fact that $u_{A, v}(z)$ is not less than the parabolic average of $u_{A, v}$ relative to an open set $G_{1}$ with compact closure in $D$, at a point $z$ of the set $G_{1}$, is proved as follows. Identifying $G_{1}$ with the set $G_{1}$ used above, we have seen that

$$
u_{A, v}(z) \geqq E\left\{f_{1}(z)\right\} .
$$

Now the conditional expectation of $f_{1}(z)$ relative to the first position at which a Brownian trajectory from $z$ meets the boundary of $D^{\prime} \cup G_{1}$ is

$$
E\left\{f_{1}\left(z_{0}\right)\right\}=u_{A, v}\left(z_{0}\right) \text {, }
$$

if this first position is $z_{0}$. Then $E\left\{f_{1}(z)\right\}$ is the parabolic average of $u_{A, v}\left(z_{0}\right)$, 
so that, by (8.5), $u_{A, v}(z)$ is not less than this average, as was to be proved.

According to the following theorem, in defining $u_{A, v}$ (with $v$ bounded) we have decreased $v$ minimally to find a function parabolic on $D^{\prime}$ and superparabolic on $D$. This fact provides a unique characterization of $u_{A, v}$.

THEOREM 8.4. Let $v$ be a function, non-negative, bounded, and superparabolic on the finite open set $D$, with stochastic boundary function 0 , and let $A$ be a subset of $D$, closed in the unextended topology. Let $u$ be a function defined on $D$, and satisfying the conditions

(a) $0 \leqq u \leqq v$;

(b) $u$ is superparabolic;

(c) $u$ is, on $D-A$, a stochastically ramified Dirichlet solution. Then $u \leqq u_{A, v}$.

Under the hypotheses, $u[v]$ has a stochastic boundary function $f^{\prime}[f]$, and, for each $z, f^{\prime}(z) \leqq f(z)$, with probability 1 , where we are supposing here that a single Brownian trajectory process is used to define both $f(z)$ and $f^{\prime}(z)$. Moreover, since $u$ is, by hypothesis, a stochastically ramified Dirichlet solution on $D-A$, we have

$$
u(z)=E\left\{f^{\prime}(z)\right\} \leqq E\{f(z)\}=u_{A, v}(z)
$$

there. If $z \in A$, and if $D-A$ is thin at $z$, we have seen above that $u_{A, v}$ and $v$ are equal at the point, so that $u \leqq u_{A, v}$ there. On the other hand, if $D-A$ is not thin at $z, z$ is a limit point of $D-A$ in the fine topology, and, since superparabolic functions are continuous in the fine topology, the desired inequality is true at $z$ since it is true on $D-A$. Thus $u \leqq u_{A, v}$.

If $A$ is an $F_{\sigma}$ subset of $D$, we can write $A=\cup_{1}^{\infty} A_{n}$, where $A_{n}$ is compact in the unextended topology, $A_{1} \subset A_{2} \subset \cdots$, and the part of $A$ in any compact (unextended topology) subset of $D$ is a subset of $A_{n}$ for sufficiently large $n$. If we apply Theorem 8.4, with $A$ replaced by $A_{n}$, and $u$ by $u_{A_{n}, v}$, we find that

Then we define

$$
v \geqq u_{A_{n+1}, v} \geqq u_{A_{n}, v} \text {. }
$$

$$
u_{A, v}=\lim _{n \rightarrow \infty} u_{A_{n}, v}
$$

The function $u_{A, v}$ is independent of the choice of the $A_{n}$ 's, is superparabolic on $D$, and Theorem 8.4 remains true. In fact, to prove Theorem 8.4 for $A$ as now defined, we need only remark that, using the restricted version of the theorem, $u \leqq u_{A_{n}, v}$, and, when $n \rightarrow \infty$ we obtain $u \leqq u_{A, v}$. In all cases it is clear that $u_{A, v}$ is parabolic on $D$ less the closure (unextended topology) of $A$.

The following example shows why this discussion must be more delicate than the corresponding discussion in the special case relevant to Laplace's equation. Let $N=1$. Let $D$ be finite $N+1$ space, let $v$ be the heat potential of a distribution on the horizontal axis, and let $A$ be this axis. Then $v$ vanishes 
identically on $A$. Hence $u_{A, v}$ would vanish identically if we used the value of $v$ on $A$ instead of the boundary limit of $v$ on $A$ (see (8.4)). In this case, $A$ is thin at each of its points. However, contrary to the possibilities in classical potential theory, $v$ may nevertheless be bounded. One obvious cause of the difficulty is that subparabolic functions need not be continuous functions of the parameter on Brownian trajectories, whereas subharmonic functions are continuous on the corresponding probability trajectories.

The function $u_{A, v}$ has the stochastic boundary function 0 , since $v$ has. If, in addition, we suppose that $v$ has the stochastic boundary function 0 in the $L_{1}$ sense, that is, if we suppose that $v$ is a potential relative to $D$ of a positive distribution on $D$, then $u_{A, v}$ will also have its boundary function 0 in the $L_{1}$ sense, and will also be a potential. We thus have a transformation taking one distribution which determines a potential into another. The new distribution is a distribution on subsets of the closure (finite topology) of $A$, because $u_{A, v}$ is parabolic on $D$ less this closure. The new distribution is said, in the classical theory, to be the result of sweeping out the old onto the closure of $A$.

We shall omit on this occasion a study of the generalization of the energy integral and its significance. This subject is made more difficult by the fact that, obviously, the Green's function of an open set is not symmetric in its two arguments.

9. Sets of absolute parabolic measure 0 . A set $A$ of $N+1$ space will be said to have absolute parabolic measure 0 if, for each finite point of $N+1$ space, almost no Brownian trajectory of any Brownian trajectory process with that initial point intersects $A$, not counting the initial point itself. In particular, if $A$ is an $F_{\sigma}$ set, this is equivalent to prescribing that $u_{A}$ vanish identically. If a set has zero absolute parabolic measure, every subset has this property. The union of a sequence of sets with this property also has the property. Every set consisting of a single finite point has this property. The set containing only the point $\infty$ does not have this property, however. If a closed set (unextended topology) has this property, it must be nowhere dense.

Let $D$ be a finite open set, and let $A$ be a subset of $D$. If, for each point of $D$, almost no Brownian trajectory of any Brownian trajectory process from that point intersects $A$ (not counting the initial point of the trajectory) before reaching the boundary of $D$, then $A$ has zero absolute parabolic measure. Hence there is no advantage to be gained in relativizing this concept. If $A$ is a closed (unextended topology) subset of $D$ it is even of zero absolute parabolic measure if $u_{A, D}$ vanishes identically on $D-A$. In fact then $u_{A, D}$ vanishes identically on $D$ because this function is superparabolic and vanishes on the set $D-A$ which scans $D$ in the technical sense of $\S 1$.

THEOREM 9.1. If $u$ is subparabolic on some open set, then the subset on which $u$ has the value $-\infty$ is a $G_{\delta}$ set of zero absolute parabolic measure. Conversely, if $I$ is a closed (unextended topology) set of zero absolute parabolic measure, there 
is a subparabolic function, with domain finite $N+1$ space, with value $-\infty$ on $A$, and finite-valued elsewhere.

If $A$ is the set where a subparabolic function has the value $-\infty$, then $A$ is a $G_{\delta}$ set, because the function is upper semicontinuous. Theorem 7.1 states in our present language that $A$ has absolute parabolic measure 0 . Conversely, let $A_{n}$ be an open set containing the part of $A$ at distance $\leqq n$ from the origin, and so small that

$$
u_{A_{n}}(z) \leqq 2^{-n}, \quad \text { if } z \in B_{n},
$$

where $B_{n}$ is the set of points at distance at least $1 / n$ from $A$. Define

$$
u=-\sum_{1}^{\infty} u_{A_{n}} \text {. }
$$

Then $u$ is finite-valued on the complement of $A$, and $-\infty$ on $A$, and $u$ is subparabolic. Presumably the full converse of this theorem is true, as it is in the special case of subharmonic functions.

If $A$ is a Borel set of $N+1$ space, it will be said to have zero heat capacity if no positive distribution of heat sources, other than the null distribution, on the Borel subsets of $A$ yields a bounded heat potential. Note that, if $A$ is below the point $z$, the potential of a non-null positive distribution is positive at $z$. Again, it is easily seen that there is no advantage in relativizing this definition.

ThEOREM 9.2. If the $F_{\sigma}$ set $A$ is of zero absolute parabolic measure, it has zero heat capacity.

If $A$ is the union of a sequence of compact sets, and if a non-null positive distribution on $A$ yields a bounded heat potential, then this distribution must be non-null on one of the summands, say $B$, and its part on $B$ will yield a bounded heat potential $u$. Let $D$ be the complement (unextended space) of $B$. Then $u$, as a bounded parabolic function on $D$, is a stochastically ramified Dirichlet solution, and therefore can be expressed on $D$ as the parabolic average of its stochastic boundary function. Since almost every Brownian trajectory meets the boundary first at $\infty$, and since $u$ vanishes below $A$, the stochastic boundary function is 0 . Hence $u$ vanishes on $D$, so the generating distribution on $D$ must be the null distribution, contrary to hypothesis.

THEOREM 9.3. Let $u$ be a subparabolic [parabolic] function on the set $D-A$, where $D$ is finite and open, and $A$ is a closed (unextended space) subset of $D$. Suppose that $u[|u|]$ is dominated by a positive stochastically ramified Dirichlet solution on $D-A$, and that $A$ has absolute parabolic measure 0 . Then $u$ can be defined on $A$ to be subparabolic [parabolic] on $D$.

According to the results of $\S 5, u$ has a stochastic boundary functicn $f$ on the boundary of $D-A$, and, in the parabolic case, $u$ is itself a stochastically 
ramified Dirichlet solution, so that, in this case,

$$
u(z)=E\{f(z)\},
$$

if $z \in D-A$. Now, according to our hypotheses, $f$ can also be considered as a stochastic boundary function of $D$, since almost no Brownian trajectory meets $A$. Hence we can use this same formula to define $u$ on $A$, and we have thus extended $u$ to be parabolic on $D$, as desired. The extension is of course unique, since $A$ is nowhere dense, so that $u$ on $A$ is uniquely determined by continuity considerations. In the subparabolic case, equality is replaced in (9.1) by inequality ( $\leqq$ ). If $u$ is defined at each point of $A$ as the value of the upper limiting function at this point, $u$ will be upper semicontinuous on $D$, and (9.1) with inequality will be true for $z \in D-A$ also, by continuity, so that $u$ will never take the value $+\infty$. Since this same inequality is true if $D$ is replaced by any open subset of itself, in particular by a standard rectangle, containing $z, u$ must be subparabolic. The function $u$ is uniquely determined on $A$ by (fine topology) continuity considerations.

10. The boundary of a set. In this section we shall discuss the set of points where a given set is thin, in particular, the set of irregular boundary points of an open set.

Theorem 10.1. Let $A$ be an $F_{\sigma}$ set of $N+1$ space. Then the set of points at which $A$ is thin is an $F_{\sigma}$ set.

In fact, let $A_{n}$ be the intersection of $A$ with the $n$th in an enumeration of the open half-spaces bounded from below by horizontal hyperplanes with rational ordinate values. Then $A$ is thin at a point $z$ if and only if it is stochastically thin there, and hence if and only if $u_{A_{n}}(z) \leqq 1 / 2$ for some $n$. The assertion of the theorem follows from the fact that each of these inequalities defines a closed set.

In the following, we shall analyze the unramified boundary of a set. Since the results are only a first step, and since other methods will probably be necessary to go further, we restrict our attention to the case $N=1$, in which the results obtained have most interest.

THEOREM 10.2. Let $N=1$, let $C$ be a finite downward directed curve of the plane, and let $D_{1}\left[D_{2}\right]$ be the domain bounded by $C$ and by the horizontal halflines with right [left] hand endpoints the initial and endpoints of $C$. Let $A_{i}$ be the set of irregular boundary points of $D_{i}$ on $C$, and let $A$ be the set of points of $C$ at which $C$ is thin. Then $A=A_{1} \cup A_{2} \cup A_{0}$, where $A_{0}$ it the set containing as its. only point the endpoint of $C$.

The endpoint of $C$ is regular for $D_{1}$ and $D_{2}$, according to the criterion of Theorem 3.1, and, on the other hand, $C$ is obviously stochastically thin and therefore thin there. If $z$ is an irregular boundary point of $D_{i}$, the complement of $D_{i}$ is thin at $z$. All the more, is the smaller set $C$ thin at $z$. Conversely, suppose that $z$ is a point of $C$, not the endpoint, at which $C$ is thin, and there- 
fore stochastically thin. This means that almost every Brownian trajectory from $z$ has an initial arc meeting $C$ only at $z$. The initial arc must lie in $D_{1}$ or $D_{2}$, aside from z. If there is positive probability that the initial arc is in $D_{i}$, the probability must be $1, z \in A_{i}$, and this finishes the proof of the lemma. Incidentally, we have proved that $A_{1}$ and $A_{2}$ have no common point.

Let $S$ be a finite downward directed curve of the plane. Then $S$ will be said to be a partial boundary of an open set $D$ on one side if $S$ is a subset of the boundary of $D$, and if each point $z$ of $S$, not an endpoint, only has points of $D$ on one side, locally, the same side for all points of $S$. We do not assume that $z$ is at positive distance from the boundary less $S$.

Let $D$ be a finite open set of $N+1$ space, with boundary $C$, and let $f$ be a boundary function in class $L(D)$. Then we have defined the function $u$, given by

$$
u(z)=E\{f[Z(z, C)]\}
$$

in $D$, as the Dirichlet solution for the heat equation. It is not really necessary, in justifying this nomenclature, to show that the irregular boundary points are irrelevant to the evaluation of $u$, but it would complete the theory in a desirable way to show this. The statement of irrelevance means that $Z(z, C)$ almost never has as value an irregular boundary point, that is, that almost no Brownian trajectory from $z$ meets $C$ in an irregular boundary point, that is, that the set of irregular boundary points has zero parabolic measure relative to $D$, identically on $D$. The simplest examples, say for $N=1$, with $D$ the interior of a circle, less a horizontal radius, show that the irregular boundary points may in fact be relevant to the evaluation of $u$, but these very examples indicate that, with a proper ramification of the boundary, the statement of the irrelevance of the irregular boundary points may still be true, and in fact we shall describe such a ramification below. The following theorem covers a case in which ramification is unnecessary. It has the advantage that it covers the classical domains always considered in discussions of parabolic equations when $N=1$. It is clear what a straightforward generalization to larger values of $N$ would be, but it is not at all clear what the most extended generalization would be.

THEOREM 10.3. Let $N=1$, and let $C$ be a downward directed curve which is $a$ partial boundary of the open set $D$ on one side. Then the set of irregular boundary points of $D$ on $C$ has parabolic measure 0 , relative to $D$, identically on $D$.

We shall use the notation of Theorem 10.2. Suppose for definiteness that $D$ is locally on the right side of $C$. Then we can and shall assume for the purposes of the proof that $C$ is finite and that $D$ contains the set $D_{2}$. Let $\{z(t)$, $0 \leqq t \leqq \infty\}$ be a Brownian trajectory process with initial point the origin. In the following, a point $\omega$ will always be a point of the measure space on which the random variables of this process are defined. Let $\Lambda(z)$ be the set corresponding to those trajectories of the process $\{z+z(t), 0 \leqq t \leqq \infty\}$, with $z \in D_{2}$, 
which meet irregular points of $C$ but no other points of this curve, and which lie in $D_{2}$, except for points of $C$, until they go below $C$. Then, if $z$ moves horizontally, it is clear that no two sets $\Lambda\left(z_{1}\right), \Lambda\left(z_{2}\right)$ have any common point. Moreover, the method of proof of Theorem 2.1 can be used to show that $P\{\Lambda(z)\}$ defines a parabolic, and therefore continuous, function of $z$. It follows that this function vanishes identically on $D_{2}$. Now if $A_{2}$ has positive parabolic measure relative to $D_{2}$ at some point $z$ of $D_{2}$, that is, if there is positive probability that a Brownian trajectory from $z$ meets the boundary of $D_{2}$ for the first time in a point of $A_{2}$, there is this same positive probability that the trajectory will meet the boundary for the first time in $A_{2}$, and then reenter $D_{2}$, for an initial arc at least, at once. Since $C$ is downward directed, once the trajectory is in $D_{2}$ again, there is positive probability that it will never meet $C$ again. But then $P\{\Lambda(z)\}$ must be positive, contrary to the above conclusion, and we can now infer that the parabolic measure of $A_{\mathbf{2}}$, relative to $D_{\mathbf{2}}$, vanishes identically on $D_{2}$. A fortiori, the parabolic measure of $A_{2}$, relative to $D$, will vanish identically on $D_{2}$. This measure even vanishes identically on $D$, as was to be proved, because, if $z \in D$, a Brownian trajectory from $z$ cannot reach the boundary first in a point of $C$ not an endpoint without passing through $D_{2}$, and we have already proved that almost no Brownian trajectory from a point of $D_{2}$ reaches the boundary first in a point of $A_{2}$. We can neglect the endpoints of $C$, because they have absolute parabolic measure 0 .

This result could be generalized by considering a domain part of whose boundary is a downward directed curve $C$, not necessarily only from one side. To handle this properly, we must count each point of $C$ for which there are (locally) points of $D$ on both sides as two points, a left and a right boundary point. In the notation of Theorem 10.2, the irregular boundary points of $D$ that are on $C$ may include right hand points, that are necessarily in $A_{2}$, and left hand points, that are necessarily in $A_{1}$. According to Theorem 10.3, almost no Brownian trajectory from a point of $D$ to the boundary, which ends in a point of $C$, and which comes in from the left [right] can end in a point of $A_{1}\left[A_{2}\right]$. In other words, in this extended sense of boundary, the irregular boundary points have parabolic measure vanishing identically on $D$. Thus the irregular boundary points on $C$ are irrelevant to our solution of the Dirichlet problem.

It is clear that the boundaries of judiciously chosen open sets of $N+1$ space, with $N>1$, can be treated in a similar way, but we leave the subject at this point, because we have not obtained any categorical results.

We now show that, if the boundary of an open set $D$ is properly ramified, the parabolic measure of the irregular boundary points of the ramified boundary vanishes identically on $D$, and that all our other results remain valid. In particular, Brownian trajectories will still almost all converge to single boundary points, and the distribution of the first meeting point of a Brownian trajectory from a point $z$ of an open set $D$ with the boundary of $D$ will be the (ramified) parabolic measure relative to $D$ at $z$. In the following, $D$ is an arbi- 
trary finite open set of $N+1$ space, with boundary $C$. Let $\mathfrak{M}$ be the space of measures of Borel subsets of $C$, with value 1 for $C$ itself, topologized in such a way that $\mu_{n} \rightarrow \mu$ if and only if, whenever $f$ is a function in the class $\hat{C}$ of functions defined and continuous on $C$, vanishing outside some bounded set,

$$
\lim _{n \rightarrow \infty} \int_{C} f d \mu_{n}=\int_{C} f d \mu
$$

The space $\mathfrak{M}$ is a separable metric space. In the following, if $z$ is a point of $C$, we denote by $m_{z}$ the element of $\mathfrak{M}$ for which the set containing this point and no other has measure 1. Let $\mu_{z}$ be the parabolic measure relative to $D$ at $z$. Then $\mu_{z}$ is a continuous function of $z$ in the topology of $\mathfrak{M}$, because, if $f$ is as above, $\int c f d \mu_{z}$ is continuous, and in fact parabolic. Moreover, if $z$ approaches a regular boundary point $w$ of $D$, it is clear that $\mu_{z}$ approaches $m_{w}$ as a limit in the topology of $\mathfrak{M}$. The key fact here is that, if $z_{0}$ is a point of $D, \mu_{z}$ has the limit $m_{w}$ in the topology of $\mathfrak{M}$, when $z$ goes to $C$ along almost every Brownian trajectory from $z_{0}$, where $w$ is the first point in which the trajectory meets $C$. This assertion is not interesting if $w$ is a regular boundary point, but the contrary case can arise with positive probability.

To prove that the assertion is true, we must prove that, for every function $f$ in the class $\hat{C}$, if $w$ is the first point in which a Brownian trajectory from $z_{0}$ meets $C$, then

$$
\lim _{z \rightarrow \infty} \int_{C} f d \mu_{s}=f(w),
$$

if $z$ approaches $w$ on the Brownian trajectory, except possibly for a set of Brownian trajectories, independent of $f$, of probability 0 . This equation, for specified $f$, is a special case of Theorem 5.2, which states that a Dirichlet solution has the given boundary function as a stochastic boundary function. The exceptional set of Brownian trajectories depends on $f$, but the desired exceptional set can be taken as the union of the exceptional sets for each of a sequence of functions $f$, where the sequence is dense (metric of uniform convergence) in the class of these functions.

The desired boundary ramification is now easily defined. We have seen that $\mu_{z}$ defines a continuous function from $D$ to $\mathfrak{M}$. There is a simplest definition of boundary of $D$ which enlarges $D$ to a complete metric space $D^{\prime}$, and makes it possible to define $\mu_{z}$ for $z \in D^{\prime}-D$ in such a way that the resulting function is continuous on $D^{\prime}$ to $\mathfrak{M}$. This is the boundary definition we use. Specifically, we consider the space of points $\left(z, \mu_{z}\right)$ for $z \in D$. We define a metric on these pairs by combining the metric of $z$ space (or a suitable extended metric if $C$ contains the point $\infty$ ) with that of $\mathfrak{M}$. This space and $D$ are homeomorphic under the correspondence $z \leftrightarrow \mu_{z}$. If we complete the space, we obtain a new metric space $D^{\prime}$, which consists of pairs $(z, \mu)$, with $z \in D \cup C$, $\mu \in \mathfrak{M}$, and certain other points. Here $\mu=\mu_{z}$ if $z \in D$, and $\mu=m_{z}$ if $z$ is a 
regular boundary point of $D$. Moreover, we have proved above that $\mu_{z}$ has a limit, in the $\mathfrak{M}$ topology, along almost every Brownian trajectory from a point of $D$ to $C$, and that this limit is the measure concentrated at the first meeting point. Thus the set of points $\left(z, m_{z}\right)$ of $D^{\prime}$, with $z \in C$, forms a subset of $D^{\prime}$ whose "projection" on $C$ is a subset of $C$ of parabolic measure identically 1 relative to $D$. The set $D$ is identified with the set of points $\left(z, \mu_{z}\right)$ in $D^{\prime}$ with the same first coordinates, and $D^{\prime}-D$ is accordingly called the ramified boundary of $D$. The ramification thus obtained leaves unaltered the regular points of $C$, but may split the irregular points. The first meeting points with $C^{\prime}$ of the Brownian trajectories from a point $z$ of $D$ are almost all of the form $\left(w, m_{w}\right)$, and the distribution of first meeting points defines a measure of Borel subsets $\mu_{z}^{\prime}$ of $C^{\prime}$. This measure will be called the ramified parabolic measure relative to $D$, at $z$. Then the discussion of the Dirichlet problem and solution proceed as usual. If $f^{\prime}$ is a function defined, bounded, and continuous on $C^{\prime}$, the corresponding Dirichlet solution, which we shall call a Dirichlet' solution, is defined by

$$
u(z)=\int_{C^{\prime}} f^{\prime} d \mu_{z}^{\prime},
$$

and, more generally, $u$ is defined by the same formula whenever $f^{\prime}$ has the property that this integral exists for all $z$ in $D$. It is clear that the class of Dirichlet' solutions is a subclass of the class of stochastically ramified Dirichlet solutions, and, in general, is a proper subclass. The two classes are the same, however, for open sets $D$ of simple structure. To prove that the set of irregular points of $C^{\prime}$ has parabolic measure 0 relative to $D$, identically on $D$, we prove that every point of $C^{\prime}$ of the form $\left(w, m_{w}\right)$ is regular. To prove this, we must show that, if $z \in D$, and if $\left(z, \mu_{z}\right)$ is near $\left(w, m_{w}\right)$, then the ramified parabolic measure at $z$ is concentrated near $\left(w, m_{w}\right)$, that is, the probability is nearly 1 that Brownian trajectories from $z$ meet $C^{\prime}$ first in points near $\left(w, m_{w}\right)$. Since almost all these trajectories meet $C^{\prime}$ in points of the form $\left(w_{0}, m_{w_{0}}\right)$, it is sufficient to show that there is probability nearly 1 that Brownian trajectories from $z$ meet $C$ first in points near $w$, and this is true because, by hypothesis, $\mu_{z}$ is near $m_{w}$.

This concludes our discussion of the ramification of the boundary of an open set. It would be natural to proceed further in this direction, by defining a ramification which would make it possible to obtain every stochastic boundary function on the original boundary from an ordinary single-valued function on the ramified boundary. It is, in fact, somewhat paradoxical that we have defined a stochastic boundary function without defining the corresponding boundary! However the ad hoc method used in the ramification just defined does not seem interesting enough to pursue further, although a purely topological approach would have considerable interest.

11. The equation $G v-\lambda v=0$. In this and later sections, when we speak of 
parabolic functions, we shall allow complex-valued ones, that is, functions whose real and imaginary parts are parabolic in the sense of the previous sections. The theorems of the previous sections will be applicable to complexvalued parabolic functions in the obvious way. We do not define complexvalued subparabolic or superparabolic functions. If $u$ is parabolic, and possibly complex-valued, $|u|$ is subparabolic.

Let $\lambda$ be a complex constant, and let $u, v$ be functions defined on an open finite set $D$ of $N+1$ space, with continuous second partial derivatives, and suppose that

$$
u(z)=e^{\lambda s} v(z),
$$$$
s=\operatorname{ord}(z) .
$$

Then $u$ satisfies the heat equation (1.1) if and only if $v$ satisfies the equation

$$
G v-\lambda v=\sum_{1}^{N} \frac{\partial^{2} v}{\partial \xi_{j}^{2}}-\frac{\partial v}{\partial s}-\lambda v=0
$$

This relationship makes it possible to treat (11.2) by referring it back to (1.1) rather than by treating it independently. The following definitions are suggested by the relationship. Let $v$ be a real function on a finite open set of $N+1$ space, and let $\lambda$ be a real constant. Then $v$ will be said to be $\lambda$ subparabolic [superparabolic] if $u$, defined by (11.1), is subparabolic [superparabolic]. Then $\lambda$ subparabolic and superparabolic functions are each other's negatives, and, if a function $v$ has continuous second partial derivatives, it is $\lambda$ subparabolic if and only if $G v-\lambda v \geqq 0$. A complex-valued function $v$ defined on a finite open set of $N+1$ space will be said to be $\lambda$ parabolic, where $\lambda$ is now complex, if $u$, defined by (11.1), is parabolic. A $\lambda$ parabolic function has continuous partial derivatives of all orders, and satisfies (11.2). If $\lambda$ and $v$ are real, $v$ is $\lambda$ parabolic if and only if it is both $\lambda$ subparabolic and $\lambda$ superparabolic. The classes of parabolic real and 0 parabolic functions coincide. If $v$ is $\lambda$ parabolic, and if $\lambda$ has real part $\lambda_{1}$, then $|v|$ is $\lambda_{1}$ subparabolic.

We can now set up a theory of Dirichlet solutions and boundary value properties of $\lambda$ subparabolic and $\lambda$ parabolic functions, but rather than doing this in detail, we shall simply state some of the typical theorems obtainable, using the correspondence (11.1), to give an idea of the results of the theory.

Let $D$ be a finite open set of $N+1$ space, with boundary $C$, and let $\lambda$ be a complex number. We shall sketch the Dirichlet solution theory for $\lambda$ parabolic functions on $D$. Suppose first that the point $\infty$ has zero parabolic measure relative to $D$, identically on $D$. Let $g$ be an ordinary boundary function, and let $\tau$ be the first time a Brownian trajectory from a point $z$ of $D$ meets the boundary. Then the Dirichlet solution for $\lambda$ parabolic functions with the specified boundary function $g$ is defined by

$$
v(z)=E\left\{e^{-\lambda r} g[Z(z, C)\} .\right.
$$

Here we suppose that the process used to define $\tau$ is the same as that used to 
define $Z(z, C)$, and that the indicated expectation exists everywhere on $D$. This condition will be satisfied, for example, if $\lambda$ has a non-negative real part; and if $g \in L(D)$. If $g$ is a stochastic boundary function, in which case $v$ will be called a stochastically ramified Dirichlet solution, the second factor in the braces is replaced by $g(z)$. With this definition, $v$ is $\lambda$ parabolic, and has $g$ as stochastic boundary function.

If the point $\infty$ may have positive parabolic measure relative to $D$, at points of $D$, the formulation is slightly different. Again, let $g$ be an ordinary boundary function, for simplicity; the extension to stochastic boundary functions is immediate. We do not suppose that $g(\infty)$ is defined, however. Instead, we shall prescribe the conduct of the Dirichlet solution at $\infty$ as will appear below. Let $\alpha$ be any complex number. Then the Dirichlet solution for $\lambda$ parabolic functions, with $g$ and $\alpha$ specified, is defined by

$$
v(z)=E\left\{e^{-\lambda \tau} g[Z(z, C)]\right\}+\alpha e^{-\lambda_{s}} p(z),
$$$$
s=\operatorname{ord}(z),
$$

where $p(z)$ is the probability that a Brownian trajectory from $z$ will meet $C$ first at $\infty$. That is, $p(z)$ is the parabolic measure of the point $\infty$, relative to $D$, at $z$. The quantity in the braces is to be taken as 0 where $\tau$ has the value $\infty$. It is supposed that $g$ and $\lambda$ are so chosen that the indicated expectation exists for all $z$ on $D$. With this definition, $v$ is $\lambda$ parabolic, has the stochastic boundary function determined by $g$, considering only finite boundary points, and $e^{\lambda \varepsilon} v(z)$ has the limit $\alpha$ along almost all Brownian trajectories from a point of $D$ which meet $C$ first at $\infty$. We have defined $v$ in such a way that, if $f(z)=e^{\lambda} g(z)$, with $f(\infty)=\alpha$, then $u$, defined by (11.1), is the Dirichlet solution for parabolic functions with boundary function $f$.

Finally, we give one example of a semimartingale theorem. Let $\lambda, v$ be real, and let $v$ be $\lambda$ subparabolic on the finite open set $D$. Suppose that the point $\infty$ has parabolic measure 0 relative to $D$, identically on $D$. Then, if $v$ is dominated from above by a positive stochastically ramified ( $\lambda$ parabolic) Dirichlet solution, $v$ has a stochastic boundary function, and, in the notation of Theorem 5.3, the process

$$
\left\{e^{-\lambda \tau_{t} v}\left[z_{z}^{*}(t)\right], 0<t \leqq \infty\right\}
$$

is a semimartingale. Here $\tau_{t}=\operatorname{Min}[\tau, t]$ and, as usual, we replace $v$ on the boundary of $D$ by the stochastic boundary function, and allow the parameter value 0 if $u(z)>-\infty$.

12. The equation $G v=1$. If $\lambda$ is a complex constant, not zero, if $v$ is a complex-valued function with continuous second partial derivatives, and if $G v=\lambda$, then $G(v / \lambda)=1$. Moreover the real part of $v / \lambda$ satisfies this same equation, and the imaginary part is parabolic. There is therefore no increase in generality in considering $v$ complex and $\lambda$ other than 1 , and we now consider the equation

$$
G v=1
$$


with $v$ real. If $v$ satisfies this equation, then

$$
G(v+s)=0, \quad G\left(v-\sum_{1}^{N} a, \xi_{j}^{2}\right)=0, \text { if } \sum_{1}^{N} a_{j}=1 / 2 .
$$

Thus (12.1) can be reduced to the heat equation in a variety of ways, and the results of our previous sections applied. We refrain from going through definitions of sub and supersolutions of (12.1), and state only one result, not as generally as we could. Let $v$ satisfy (12.1) on an open set $D$ of $N+1$ space. Let $D$ be bounded from below. Suppose that $g$ is a function defined on the boundary $C$ of $D$, and is in the class $L(D)$. Let $z(t), \tau, \tau_{t}$, be defined as in the preceding section. Then the function $v$ defined by

$$
v(z)=E\{g[Z(z, C)]-\tau\}, \quad z \in D,
$$

satisfies (12.1), and the process $\left\{v\left[z\left(\tau_{t}\right)\right]-\tau_{t}, 0 \leqq t \leqq \infty\right\}$ is a martingale. The function $v$ satisfies (12.1) and has the specified boundary function $g$ as a limit along almost all Brownian trajectories from a point of $D$, that is, $v$ has a stochastic boundary function, that determined by $g$.

13. $\lambda$ subharmonic and $\lambda$ harmonic functions. Let $u$ be $\lambda$ subparabolic [parabolic] on a finite open set of $N+1$ space, and let this set $D$ be the direct product of an open set $D_{0}$ in $N$ space and the $s$ axis. Suppose also that $u$ does not depend on $s$. Then $u$ defines a function $u_{0}$ on $D_{0}$. The function $u_{0}$ will be called $\lambda$ subharmonic [harmonic] on $D_{0}$. A function is 0 harmonic if and only if its real and imaginary parts are harmonic. It is 0 subharmonic if and only if it is real and subharmonic in the usual sense. If $u_{0}$ has continuous second partial derivatives, it is $\lambda$ subharmonic if and only if it and $\lambda$ are real, and if

$$
\sum_{1}^{N} \frac{\partial^{2} \mu_{0}}{\partial \xi_{i}^{2}} \geqq \lambda u_{0} \text {. }
$$

The $\lambda$ subharmonic functions can also be defined in a way reminiscent of one standard definition of subharmonic functions. A real function $u_{0}$ is $\lambda$ subharmonic on an open set of $N$ space if and only if it satisfies the following conditions, where we write $\xi$ for $\left(\xi_{1}, \cdots, \xi_{N}\right)$ :

(i) $-\infty \leqq u_{0}<+\infty$, and $u_{0}(\xi) \not \equiv-\infty$;

(ii) $u_{0}$ is upper semicontinuous;

(iii) if $u_{R}(\xi)$ is the average of $u_{0}$ over the surface of a sphere with center $\xi$, of radius $R$, then, for sufficiently small $R, \alpha_{N, \lambda}(R) u_{0}(\xi) \leqq \mu_{R}(\xi)$, where

$$
\alpha_{N, \lambda}(R)=\frac{\Gamma(N / 2) J_{(N-2) / 2}\left(R(-\lambda)^{1 / 2}\right)}{\left(r(-\lambda)^{1 / 2} / 2\right)^{(N-2) / 2}} .
$$

The function $\alpha_{N, \lambda}$ is the unique solution of the differential equation

$$
\alpha^{\prime \prime}+\frac{N-1}{R} \alpha^{\prime}=\lambda \alpha
$$


which is an entire function, and for which $\alpha(0)=1$. In particular, when $\lambda \rightarrow 0$, we find the limiting case of subharmonic functions, with $\alpha_{N, 0}(R)=1$.

For arbitrary complex $\lambda \neq 0$, if a function $v$ is $\lambda$ harmonic on a set of $N$ space, then $v \exp \left((-\lambda)^{1 / 2} \xi_{N+1}\right)=v_{1}$ is 0 harmonic in $N+1$ dimensions. Here $(-\lambda)^{1 / 2}$ is either root of $-\lambda$. It follows that a $\lambda$ harmonic function is an analytic function of its arguments. If $\lambda$ is negative and if $v$ is real, then $v$ is $\lambda$ subharmonic if and only if $v_{1}$ is subharmonic. If $\lambda$ is positive, we use the same idea. The function $\alpha_{N, \lambda}$ defines a parabolic function of $R=\left(\sum_{1}^{N} \xi_{j}^{2}\right)^{1 / 2}$. Then it follows that if $v$ is real it is $\lambda$ subharmonic if and only if

$$
v(\xi) \alpha_{2,-\lambda}\left[\left(\xi_{N+1}^{2}+\xi_{N+2}^{2}\right)^{1 / 2}\right]
$$

defines s subharmonic function in $N+2$ dimensions.

The Brownian trajectories of interest in studying $\lambda$ subharmonic and harmonic functions are of course the projections of the Brownian trajectories used above onto the horizontal hyperplane through the origin. That is, the trajectories of interest are the sample curves of the $N$ dimensional Brownian motions. We now show that, if $v$ is $\lambda$ subharmonic on an open set of $N$ space, it is continuous, as a function of the parameter, along almost all such trajectories from a point. Theorem 7.2, as applied to the function $e^{\lambda \boldsymbol{\theta}} \boldsymbol{v}_{\text {, only implies }}$ right continuity, but the full result can be obtained by reducing the general case to the case $\lambda=0$, where the result is known [2]. This can be done by using the above correspondence between $\lambda$ subharmonic and subharmonic functions.

We omit a discussion of the Dirichlet problem and associated boundary value problems for $\lambda$ harmonic and subharmonic functions. In fact, the theorems follow at once from the more general theorems on $\lambda$ parabolic and $\lambda$ subparabolic functions. The criterion of irregularity of a boundary point for the Dirichlet problem can be obtained from Theorem 3.1 by replacing the Brownian trajectories in $N+1$ dimensions by the Brownian motion sample curves in $N$ dimensions, in other words by projecting the trajectories into $N$ space. The study of $\lambda$ subharmonic and $\lambda$ harmonic functions is greatly simplified by two facts. (a) For every real $\lambda$, as we have shown above, $\lambda$ subharmonic functions are continuous, as functions of the parameter, along Brownian motion curves. (b) For all complex $\lambda$, the set of irregular boundary points of an open set, relevant to the Dirichlet problem for $\lambda$ harmonic functions, even without a ramification of the boundary, is irrelevant to a solution of the Dirichlet problem. In other terms, almost no $N$ dimensional Brownian motion sample curve from a point of $N$ space ever passes through an irregular point of an open set. (See [2] for proofs for the case $\lambda=0$ and further references.)

14. Distribution of first passage time. We now apply the results of the preceding sections to find the distribution of the time it takes a Brownian trajectory from a point to meet a specified closed set. We shall formulate the 
problem as follows. Let $D$ be a finite open set of $N+1$ space, and let $\tau$ be the time a Brownian trajectory from a point $z$ of $D$ first meets the boundary (first passage time). To avoid eyestrain, we omit $z$ from the first passage time notation, but $\tau$ is a random variable, for each initial point $z$, perhaps defined on different measure spaces for different values of $z$. The family of all these random variables is not a stochastic boundary function, but if, for each $z$, we take the random variable $s-\tau$, where $s$ here and in the following is always $\operatorname{ord}(z)$, the family of random variables thus defined is a stochastic boundary function. We shall now exhibit two methods of finding the distribution of $\tau$. These two methods are actually only one method from two points of view, but the different points of view are rather suggestive.

Let $\lambda$ be a complex number. Then $e^{\lambda s}$ is a boundary function of $D$; we define the exponential as 0 at the point $\infty$. Suppose that this boundary function is in the class $L(D)$. This will be true, for example, if $D$ is bounded from below, or if $\tau$ has a non-negative real part. Define $u_{\lambda}$ as the Dirichlet solution for parabolic functions with this boundary function,

$$
u_{\lambda}(z)=E\left\{e^{\lambda(s-r)}\right\}=e^{\lambda \varepsilon} E\left\{e^{-\lambda r}\right\},
$$

defining the exponential as 0 where $\tau$ has the value $+\infty$. Thus the knowledge of $u_{\lambda}$, which is obtained by solving a Dirichlet problem, that is, by solving the differential equation $G u=0$ with the given boundary conditions (and imposing enough conditions to ensure that $u$ is actually the Dirichlet solution) serves to identify the distribution of $\tau$. In fact, one can take $\lambda$ real and positive, and find the Laplace transform of the distribution. Or, if $\tau$ is almost certainly finite, one can take $\lambda$ pure imaginary, and find the characteristic function of the distribution of first passage time.

Alternatively, if one can solve the Dirichlet problem for $\lambda$ parabolic functions on $D$, with boundary function 1 , taking as 0 the constant governing the behavior at $\infty$ (see $\$ 11)$, one obtains a function $v_{\lambda}$,

$$
v_{\lambda}(z)=E\left\{e^{-\lambda \tau}\right\} .
$$

Of course the two problems are equivalent, and $u_{\lambda}=e^{\lambda} v_{\lambda}$.

The procedure is now clear. Given any boundary function, a solution of the Dirichlet problem gives information on the distribution of first passage time. As a second example, we consider the problem of evaluating the expected passage time, assuming that $E\{\tau\}$ is finite. If $u$ is the solution on $D$ of the Dirichlet problem for parabolic functions, with boundary function $s$, then

$$
u(z)=E\{s-\tau\}=s-E\{\tau\} .
$$

Alternatively, if $v$ is the Dirichlet solution on $D$ of the Dirichlet problem for the equation $G u=1$, with boundary function 0 , then

$$
v(z)=-E\{\tau\}=u(z)-s .
$$

Note that, even if the boundary function is continuous, in finding these 
Dirichlet solutions it is not enough to find functions satisfying the specified differential equations and assuming the given boundary values at the regular boundary points, because these conditions may not determine the function uniquely, that is, as the Dirichlet solution with the specified boundary function.

We illustrate the problem with an example. Suppose that we wish to find the function $u_{\lambda}$ above, with $\lambda$ real and positive. Then $u_{\lambda}$ must be bounded on the part of $D$ below any horizontal hyperplane. Suppose that a parabolic function $w_{\lambda}$ is found, with boundary limit $e^{-\lambda_{s}}$ at all the regular boundary points of $D$, and bounded on the part of $D$ below every horizontal hyperplane. Then we know from Theorem 5.3 that $w_{\lambda}$ is a stochastically ramified Dirichlet solution, but we do not know that it is the correct one unless we know that the irregular boundary points can be ignored, that is, that they form a set of parabolic measure 0 relative to $D$, identically on $D$. In most simple cases this is obviously true, at least if the boundary is ramified in the obvious way. For example, according to our work, it is true if $N=1$ and if the boundary of $D$ is the union of at most denumerably many downward directed curves and horizontal line segments, only a finite number of which meet any bounded set.

The problem is simpler if one can deal with $\lambda$ harmonic functions, that is if the domain $D$ is the direct product of an open set $D_{0}$ in $N$ dimensions, and the $s$ axis, since in that case the set of irregular boundary points of $D$ always has absolute parabolic measure 0 because the set of irregular boundary points of $D_{0}$ has absolute harmonic measure 0 . As a simple example of the use of $\lambda$ harmonic functions, let $D_{0}$ be a sphere in $N$ space, center the origin, radius $R$. Then, if $\lambda$ has a non-negative real part,

$$
E\left\{e^{-\lambda \tau}\right\}=\frac{\alpha_{N, \lambda}(|\xi|)}{\alpha_{N, \lambda}(R)}, \quad|\xi|=\left(\sum_{1}^{N} \xi_{j}^{2}\right)^{1 / 2}
$$

where $\alpha_{N, \lambda}$ was defined in $\S 13$. In fact, if $v$ is the solution of the Dirichlet problem for $\lambda$ harmonic functions on $D_{0}$, with boundary function 1 ,

$$
v(\xi)=E\left\{e^{-\lambda \tau}\right\},
$$

according to the results in $\$ 13$, that is, according to the general results for $\lambda$ parabolic functions, reduced to the $\lambda$ harmonic case by projection. The function $v$ is $\lambda$ harmonic on $D_{0}$, and, if defined as 1 on the boundary is continuous on the closure of $D_{0}$, since every boundary point is regular. The right side of (14.1) is also $\lambda$ harmonic on $D$, continuous on the closure of $D$, and is therefore the Dirichlet solution with boundary function 1, the value on the boundary. Thus this function is $v$, as was to be proved. We have restricted ourselves by choosing $\lambda$ to have a non-negative real part, to make certain that $v$ would be well defined.

In investigations of first passage times, it is frequently not known in ad- 
vance whether the required expectations exist. The following example illustrates how these methods can be adapted under such circumstances, by applying the general theorems to properly chosen subsets of $D$. Suppose that $D$ lies in the strip between two parallel hyperplanes which are parallel to the $s$ axis. That is, there is a linear combination $h$ of the space coordinate functions which is bounded on $D$. We can suppose that $h$ has the form

$$
h(z)=\sum_{1}^{N} a_{j} \xi_{j}, \quad \sum_{1}^{N} a_{j}^{2}=1 .
$$

In this case, the first passage time is finite, with probability 1 , whatever the initial point. Let $c$ be a real constant. The function

$$
u=e^{i c h-c^{2} s}
$$

is parabolic on $N+1$ space, and is bounded above every horizontal hyperplane. Hence $u$ is the Dirichlet solution for parabolic functions on $D$ with its own values on the boundary determining the boundary function, if $D$ is bounded from below,

$$
u(z)=E\left\{e^{i c h[z(r)]-c^{2}(r-\tau)}\right\} .
$$

Now $h$ is bounded on $D$, by hypothesis, so we can choose $|c|$ so small that $|c h|<\pi / 3$ on the closure of $D$. With $c$ chosen in this way, and if $D$ is bounded from below,

$$
|u(z)| \geqq 2^{-1} e^{-c^{2} z} E\left\{e^{c^{2} \tau}\right\} .
$$

This inequality must hold even if $D$ is not bounded from below because it holds for bounded subsets of $D$. Hence $E\left\{e^{c^{2} \tau}\right\}$ is finite, if $|c|$ is chosen small, as described above. It now follows that, for $c$ so chosen, since (14.2) is true for bounded subsets of $D$, it must be true for $D$ itself. The finiteness of $E\left(e^{c^{2} r}\right\}$ could also have been obtained from the distribution of $\tau$ for $N=1$ when $D$ is the strip between two lines parallel to the $s$ axis, in which case there are explicit formulas available for the distributions involved. We now continue this example, and show, always under the hypothesis that $h$ is bounded on $D$, that $E\left\{e^{c|z(r)|}\right\}$ is finite, for $c$ positive and sufficiently small. If $N=1$, this follows from the fact that then $|z(\tau)|$ is of the order of magnitude of $\tau$, for large $\tau$. In the general case, it is sufficient to show that, if $\xi_{j}(z)$ is the $j$ th coordinate of the point $z$, if $b$ is real and sufficiently near 0 , and if $j \leqq N$, then

$$
E\left\{e^{b \xi_{j}[z(\tau)]}\right\}<\infty \text {. }
$$

The function

$$
e^{b \xi_{j}(z)+b^{2}}
$$

is parabolic, and hence, if $D$ is bounded, is the Dirichlet solution on $D$, with its values on the boundary determining the boundary function, 


$$
e^{b \xi_{j}(z)}=E\left\{e^{b \xi_{j}[z(r)]-b^{2} r}\right\} .
$$

If $D$ is not bounded, the equation is true for bounded subsets of $D$, and hence taking an expanding sequence of such sets, we find that, for $D$ itself,

$$
e^{b \xi_{j}(z)} \geqq E\left\{e^{b \xi_{j}[z(r)]-b^{2} r}\right\} .
$$

Hence, applying Schwarz's inequality,

$$
E\left\{e^{b \xi_{j}[z(\tau)]}\right\} \leqq e^{b \xi_{j}} E^{1 / 2}\left\{e^{4 b^{2} \tau}\right\},
$$

and we have seen that the right side of this inequality is finite if $|b|$ is sufficiently small. This finishes our proof.

With this same domain $D$, we now use the fact that $2 N s+\xi_{j}(z)^{2}$ is parabolic, and defines a boundary function in the class $L(D)$, according to what we have just proved. Hence

$$
2 N s+\xi_{j}(z)^{2}=E\left\{2 N s-2 N \tau+\xi_{j}[z(\tau)]^{2}\right\}
$$

so that

$$
E\{\tau\}=\frac{1}{2 N} E\left\{\xi_{j}[z(\tau)]^{2}\right\}-\frac{1}{2 N} \xi_{j}(z)^{2} .
$$

In particular, if $D$ is a cylinder, with axis the $s$ axis, and radius $R$, so that we are really dealing with the first passage time of a Brownian motion in $N$ dimensions, with domain a sphere with center the origin and radius $R$, and if we average these equations over $j$, we obtain

$$
E\{\tau\}=\frac{R^{2}}{2 N^{2}}-\frac{1}{2 N^{2}} \sum_{1}^{N} \xi_{j}(z)^{2} .
$$

\section{BIBLIOGRAPHY}

1. J. L. Doob, Stochastic processes, New York, 1953.

2. - Semimartingales and subharmonic functions, Trans. Amer. Math. Soc. vol. 77 (1954) pp. 86-121.

3. - Martingales and one-dimensional diffusion, Trans. Amer. Math. Soc. vol. 78 (1955) pp. 168-208.

4. Robert Fortet, Les fonctions aléatoires du type de Markoff associees à certaines equations linéaires aux dérivées partielles du type parabolique, J. Math. Pures Appl. vol. 22 (1943) pp. 177243.

5. P. Hartman and A. Wintner, On the solutions of the equation of heat conduction, Amer. J. Math. vol. 72 (1950) pp. 367-395.

6. I. Petrowsky, Zur ersten Randwertaufgabe der Wärmeleitungsgleichung, Compositio Math. vol. 1 (1935) pp. 383-419.

7. D. V. Widder, Positive temperatures on an infinite rod, Trans. Amer. Math. Soc. vol. 55 (1944) pp. 85-95.

UNIVERSITY OF ILLINOIS, URBANA, ILL. 